TRANSACTIONS OF THE

AMERICAN MATHEMATICAL SOCIETY

Volume 361, Number 3, March 2009, Pages 1209-1240

S 0002-9947(08)04638-2

Article electronically published on October 23, 2008

\title{
SPECTRAL CLUSTER ESTIMATES FOR METRICS OF SOBOLEV REGULARITY
}

\author{
MATTHEW D. BLAIR
}

\begin{abstract}
We investigate spectral cluster estimates for compact manifolds equipped with a Riemannian metric whose regularity is determined by its inclusion in a Sobolev space of sufficiently high order. The problem is reduced to obtaining $L^{p}$ estimates for the wave equation which are shown by employing wave packet techniques.
\end{abstract}

\section{INTRODUCTION}

Spectral cluster estimates refer to a family of inequalities on eigenfunctions of the Laplacian on a compact Riemannian manifold $(M, g)$. It is well known that the Laplace-Beltrami operator associated to $(M, g)$, denoted by $\Delta_{g}$, possesses a sequence of eigenfunctions $\left\{\phi_{j}\right\}_{j=0}^{\infty}$ that form an orthonormal basis for $L^{2}\left(M, d V_{g}\right)$, $d V_{g}$ denoting the Riemannian measure induced by $g$. We take the convention that the eigenfunctions are ordered so that

$$
\Delta_{g} \phi_{j}=-\lambda_{j}^{2} \phi_{j} \text { with } 0=\lambda_{0} \leq \lambda_{1} \leq \cdots \leq \lambda_{j} \leq \lambda_{j+1} \leq \cdots .
$$

We define the spectral projection operator $\Pi_{\lambda}$ for any $\lambda \geq 0$ as

$$
\Pi_{\lambda} f=\sum_{\lambda_{j} \in[\lambda, \lambda+1]}\left\langle f, \phi_{j}\right\rangle \phi_{j} .
$$

Spectral cluster estimates then state that

$$
\left\|\Pi_{\lambda} f\right\|_{L^{q}(M)} \leq C \lambda^{\delta(q)}\|f\|_{L^{2}(M)}, \quad \delta(q)= \begin{cases}\frac{n-1}{2}\left(\frac{1}{2}-\frac{1}{q}\right), & 2 \leq q \leq q_{n}, \\ n\left(\frac{1}{2}-\frac{1}{q}\right)-\frac{1}{2}, & q_{n} \leq q \leq \infty,\end{cases}
$$

with $q_{n}=\frac{2(n+1)}{n-1}$ and $C$ independent of $\lambda$. These inequalities were originally developed by C.D. Sogge in 13 for compact Riemannian manifolds with $C^{\infty}$ metrics.

It is also of interest to investigate whether or not such estimates hold for manifolds equipped with rougher metrics of limited differentiability. The first work in this direction came in [11, where H. Smith and C.D. Sogge provided examples of $C^{1, \alpha}$ metrics for each $\alpha \in[0,1)$ where the spectral cluster estimates fail to hold in the range $2 \leq q \leq q_{n}$. Other examples are in [12]. The natural question then was whether or not such estimates should hold for $C^{1,1}$ metrics. Indeed, the geodesic flow is well-behaved for such metrics, suggesting that such estimates should hold.

Recently, in [10, H. Smith proved these estimates for $C^{1,1}$ metrics by employing wave equation methods. The main idea here is that by examining the effect of the

Received by the editors August 28, 2006.

2000 Mathematics Subject Classification. Primary 42C15; Secondary 35P99, 35L15, 35R05.

The author was supported in part by the NSF grant DMS-0354668.

(C)2008 American Mathematical Society 1209

Reverts to public domain 28 years from publication 
wave group on a spectral cluster $\Pi_{\lambda} f$ one can reduce the estimates (1.1) to certain mixed $L^{q}$ estimates on solutions to the wave equation. These inequalities, often called "squarefunction estimates" state that if $u(x, t): M \times\left[-t_{0}, t_{0}\right] \rightarrow \mathbb{C}$ denotes a solution to the wave equation and $\infty \geq q \geq q_{n}$, then

$\|u\|_{L_{x}^{q}\left(M ; L_{t}^{2}\left[-t_{0}, t_{0}\right]\right)} \leq C\left(\left\|\left(u(0, \cdot), \partial_{t} u(0, \cdot)\right)\right\|_{H^{\delta(q)} \times H^{\delta(q)-1}}+\|P u\|_{L_{t}^{1}\left(\left[-t_{0}, t_{0}\right] ; H^{\delta(q)-1}\right)}\right)$, with $\delta(q)$ defined as above. Here $P=\partial_{t}^{2}-\Delta_{g}$ denotes the wave operator and $H^{s}$ denotes the $L^{2}$ Sobolev space of order $s$ over $M$ with $\delta(q)$ as in (1.1). Squarefunction estimates were first developed for $C^{\infty}$ metrics by Mockenhaupt, Seeger, and Sogge in [6]. They were then shown in the case of $C^{1,1}$ metrics in the aforementioned work of Smith [10.

To handle metrics rougher than $C^{1,1}, \mathrm{H}$. Smith then developed a family of weaker spectral cluster estimates for $C^{1, \alpha}$ metrics (see [9]). These estimates bound the operator norm of $\Pi_{\lambda}$ as a map from $L^{2}(M)$ to $L^{q}(M)$ by a power of $\lambda$ higher than that appearing in (1.1). Specifically, if $g$ is a $C^{1, \alpha}$ metric with $0 \leq \alpha \leq 1$ and $\sigma=\frac{1-\alpha}{3+\alpha}$, then his results show that

$$
\begin{aligned}
& \left\|\Pi_{\lambda}\right\|_{L^{2}(M) \rightarrow L^{q}(M)} \leq C \lambda^{\delta(q)(1+\sigma)}, \quad 2 \leq q \leq \frac{2(n+1)}{n-1}, \\
& \left\|\Pi_{\lambda}\right\|_{L^{2}(M) \rightarrow L^{q}(M)} \leq C \lambda^{\delta(q)+\frac{\sigma}{q}}, \quad \frac{2(n+1)}{n-1} \leq q \leq \infty .
\end{aligned}
$$

The examples of [11, 12] show that such estimates are sharp for $q$ in the range $2 \leq$ $q \leq q_{n}$. We also remark that recent work of Koch-Smith-Tataru [5] has developed a version of these estimates for Hölder $C^{s}$ metrics below Lipschitz regularity which are sharp for $2 \leq q \leq q_{n}$ and $q=\infty$.

The estimates (1.3), (1.4) are equivalent to a weaker version of the squarefunction estimates for wave equations in $C^{1, \alpha}$ metrics which state that

$$
\begin{aligned}
& \|u\|_{L_{x}^{q}\left(M ; L_{t}^{2}\left[-t_{0}, t_{0}\right]\right)} \\
& \quad \leq C\left(\left\|\left(u(0, \cdot), \partial_{t} u(0, \cdot)\right)\right\|_{H^{\delta(q)+\frac{\sigma}{q}} \times H^{\delta(q)+\frac{\sigma}{q}-1}}+\|P u\|_{L_{t}^{1}\left(\left[-t_{0}, t_{0}\right] ; H^{\delta(q)+\frac{\sigma}{q}-1}\right)}\right) .
\end{aligned}
$$

Hence there is an increase in the number of derivatives required on the right hand side. These estimates follow from (1.4), but can also be shown directly by adapting the "truncation/rescaling" methods for Strichartz estimates found in the work of D. Tataru (see [16, 17, [15]) and in Bahouri-Chemin (see [1, 2]). Their results demonstrated that a weaker version of the related Strichartz estimates hold, which control the $L_{t}^{p} L_{x}^{q}$-norm of solutions to wave equations with $C^{s}$ coefficients when $0<s \leq 2$.

The purpose of this paper is to consider spectral cluster estimates in the case where the metric tensor $g$ lies in an $L^{r}$ Sobolev space of sufficiently high order with $1<r<\infty$. Specifically, we seek to establish the estimates (1.1) for metrics in $L^{r, \kappa}$ (the $L^{r}$ Sobolev space of order $\kappa$ ) for $\kappa>\frac{n-1}{r}+2$. In addition, we use truncation/rescaling methods to show that the estimates (1.3), (1.4) hold when the metric lies in $L^{r, \kappa} \cap L i p$ with $\kappa=\frac{n-1}{r}+1+\alpha$ for $\alpha \in(0,1)$ and $\sigma=\frac{1-\alpha}{3+\alpha}$ determined by the $\alpha$ appearing in the definition of $\kappa$.

Clearly, the property $g \in L^{r, \kappa}(M), \kappa>\frac{n-1}{r}+2$, does not imply that the metric tensor is $C^{1,1}$. However, there is enough differentiability to ensure that the geodesic flow exists and is well-behaved. These properties were used in a recent work by the author (see [3]) to show that in dimensions $n=2,3$, the Strichartz estimates hold for solutions to wave equations whose coefficients possess this degree of Sobolev 
regularity. Strichartz estimates are similar to the squarefunction inequalities in (1.2) in that they control mixed $L^{p}$ norms of the solution. However, they are distinct in that they depend largely on the dispersive effect of the solution operator, whereas squarefunction inequalities also rely on the decay of the operator away from the light cone. Nevertheless, a high degree of control of the geodesic flow is important in establishing both sets of inequalities. At the moment, we refer the reader to section 1 and section 4 of 3 for a detailed discussion of these ideas in the context of Strichartz estimates, though they will also appear below.

Metrics possessing this degree of Sobolev regularity arise naturally if one looks only at $L^{r}$ bounds on the Ricci tensor. If one assumes a mild degree of regularity on the metric $g$, it can be shown that the manifold can be covered by harmonic coordinate charts, that is, coordinates $\left(y_{1}, \ldots, y_{n}\right)$ such that $\Delta_{g} y_{l}=0$ for each coordinate function $y_{l}$. See Proposition 9.1 and the surrounding discussion in Chapter 3 of [20] for an approach that only requires $g \in C^{s}(M)$. In such coordinates, it can be shown that the metric satisfies a quasilinear elliptic system of equations

$$
\partial_{j} g^{j k}(y) \partial_{k} g_{l m}(y)+Q_{l m}(g, \nabla g)=R i c_{l m}
$$

where $Q_{l m}(g, \nabla g)$ is a quadratic form in $\nabla g$ with coefficients determined by $g$. By applying elliptic regularity results for such systems (see Corollary 12B.5 in Chapter 14 of [18] and Proposition 10.1 of [20]), we have that if $p \geq \frac{n}{2}, 1<r<\infty$, and $\kappa \geq 2$, then

$$
g_{j k} \in H^{1}\left(B_{1}\right) \cap C^{0}\left(B_{1}\right), R i c_{l m} \in L^{r, \kappa-2}\left(B_{1}\right) \cap L^{p}\left(B_{1}\right) \Rightarrow g_{j k} \in L^{r, \kappa}\left(B_{\frac{1}{2}}\right),
$$

with $B_{r}$ denoting a ball of radius $r$ in the coordinate chart. See Proposition 4.10 in Chapter 14 of [18] for another result of this type which requires less regularity from the Ricci tensor. Hence our results will provide a weaker form of the spectral cluster estimates (1.5) for " $L^{r}$-pinched" metrics whose Ricci tensor (defined in the sense of distributions) satisfies Ric $\in L^{r}(M), r \geq \frac{n}{2}$, and $\alpha$ satisfying $\frac{n-1}{r}+1+\alpha=2$.

Finite speed of propagation allows one to work in coordinate charts and easily reduce the estimates (1.2), (1.5) to establishing squarefunction inequalities over $\mathbb{R}^{n}$ for solutions to the Cauchy problem with $\rho(x)=\sqrt{\operatorname{det}\left(g_{i j}\right)}, a_{i j}(x)=g^{i j}(x) \rho(x)$ :

$$
\begin{gathered}
P(x, D) u(x, t):=\rho(x) \partial_{t}^{2} u(x, t)-\sum_{1 \leq i, j \leq n} \partial_{x_{i}}\left(a_{i j}(x) \partial_{x_{j}} u(x, t)\right)=F(x, t), \\
u(x, 0)=f, \partial_{t} u(x, 0)=g .
\end{gathered}
$$

One of the main challenges in proving estimates for wave equations in this context lies in constructing a suitable parametrix for the equation. When the coefficients of $P$ are smooth, there are asymptotic methods available for this purpose, but are inapplicable in the non-smooth setting. An effective substitute is to use wave packets in constructing a parametrix. In our context, wave packets are approximate solutions to the wave equation that are highly localized in space and in frequency. They can be used to construct an effective parametrix for the wave equation by writing general solutions as a superposition of these packets. The first work in this direction is that of Smith [8, who found a parametrix that represented solutions as a discrete sum of travelling wave packets. This gave Strichartz estimates in dimensions $n=2,3$ for wave operators with $C^{1,1}$ coefficients. In [15, [16], 17, Tataru then followed in a similar vein, using the Fourier-Bros-Iagolnitzer (FBI) transform in space-time to construct a parametrix that instead wrote solutions as a continuous superposition of wave packets. This approach showed that the Strichartz 
inequalities hold in all dimensions for operators with $C^{1,1}$ coefficients (as well as operators with coefficients satisfying (1.7) below).

Squarefunction estimates for wave equations with rough coefficients then came in [10. In this work, Smith constructed a parametrix by taking a continuous wave packet transform in the spatial variables. This approach amounted to representing solutions as a continuous superposition of travelling wave packets. The nature of this transform was quite similar to the wave packets of Cordoba-Fefferman 4 as well as the FBI transform used by Tataru. This parametrix provided an effective way of representing solutions to equations with time-dependent coefficients satisfying

$$
\partial_{x, t}^{\beta} \rho(x, t), \partial_{x, t}^{\beta} a_{i j}(x, t) \in L_{t}^{1}\left(\left[-t_{0}, t_{0}\right], L_{x}^{\infty}\left(\mathbb{R}^{n}\right)\right), \quad|\beta| \leq 2 .
$$

These techniques are not immediately applicable when coefficients possess the Sobolev regularity outlined above. Indeed, the ability to control the $L^{\infty}$-norm of the second order derivatives of the coefficients over all spatial variables plays a crucial role in the constructions mentioned above. Therefore, a different perspective on the problem is needed. Suppose for now that $\|\rho-1\|_{L^{\infty}},\left\|a_{i j}-\delta_{i j}\right\|_{L^{\infty}} \leq \frac{1}{4}$ so that $P$ is in some sense a perturbation of the constant coefficient wave operator. Write the principal symbol of $-P(x, \xi, \tau)$ as

$$
a_{11}(x) \xi_{1}^{2}+2 \sum_{j=2}^{n} a_{1 j}(x) \xi_{1} \xi_{j}-\left(\rho(x) \tau^{2}-\sum_{2 \leq i, j \leq n} a_{i j}(x) \xi_{i} \xi_{j}\right) .
$$

We now see that $P$ can be viewed as an operator that is hyperbolic in $x_{1}$ when restricted to space-time frequencies lying in a set of the form

$$
\left\{(\xi, \tau):|\tau| \gg\left|\left(\xi_{2}, \ldots, \xi_{n}\right)\right|\right\}
$$

since this means that the principal symbol of $P$ is a quadratic in $\xi_{1}$ with 2 distinct real roots. But if the coefficients lie in $L^{r, \kappa}$ with $\kappa>\frac{n-1}{r}+2$, Sobolev embedding implies that for any $T$ in the orthogonal group $O(n)$ and any multi-index $|\beta| \leq 2$,

$$
\partial_{x}^{\beta}\left(\left(a_{i j} \circ T\right)(x)-\delta_{i j}\right) \in L_{x_{1}}^{r}\left(\mathbb{R} ; L_{x^{\prime}}^{\infty}\left(\mathbb{R}^{n-1}\right)\right) \subset L_{x_{1}, l o c}^{1}\left(\mathbb{R} ; L_{x^{\prime}}^{\infty}\left(\mathbb{R}^{n-1}\right)\right),
$$

where $x=\left(x_{1}, x^{\prime}\right)$. Here the latter set is the space of locally integrable functions in the $x_{1}$ variable with values in the Banach space of $L^{\infty}$ functions over the $x^{\prime}$ variable. When $T$ is the identity, this is akin to (1.7) for operators hyperbolic in $x_{1}$. This suggests that squarefunction estimates should hold for solutions to the wave equation that are localized to frequencies in the set (1.8). It should then be possible to prove squarefunction estimates for arbitrary solutions to the wave equation by representing them as a sum of solutions localized in frequency to regions of this type. Indeed, it was this perspective that allowed for a proof of the Strichartz estimates in [3] and will now form the backbone of our arguments here.

Our main approach will be to combine the frequency localization arguments of [3] with the wave packet transform techniques of [10. We first reduce the problem to showing estimates on components of our solution that are localized in frequency to dyadic regions contained in (1.8). Once this is done, we show that these components are also solutions to a first-order pseudodifferential equation that is hyperbolic in $x_{1}$. With these reductions in place, we are then able to use a wave packet transform as in Smith 10 to represent these localized functions as a continuous superposition of wave packets parameterized by $x_{1}$. It is then a matter of showing that this parametrix yields the desired $L_{x}^{q} L_{t}^{2}$ estimates. 
We will also prove squarefunction estimates for coefficients in the space $L^{r, \kappa}$, $\kappa=\frac{n-1}{r}+1+\alpha$. Such coefficients satisfy the property that for any $T$ in the orthogonal group $O(n)$ and any multi-index $|\beta| \leq 1$,

$$
\partial_{x}^{\beta}\left(\left(a_{i j} \circ T\right)(x)-\delta_{i j}\right) \subset L_{x_{1}, l o c}^{1}\left(\mathbb{R} ; C_{x^{\prime}}^{1-|\beta|, \alpha}\left(\mathbb{R}^{n-1}\right)\right) .
$$

This property will allow us to use elements of the previously mentioned "truncation/rescaling" approach to prove inequalities of the form (1.5).

The main results of this paper are the following:

Theorem 1.1. Assume that $\rho-1, a_{i j}-\delta_{i j} \in L^{r, \kappa}\left(\mathbb{R}^{n}\right)$, with $\kappa>\frac{n-1}{r}+2$, and that $0 \leq \delta(q) \leq \frac{3}{2}$. Then there exists $C$ depending only on the time interval, the dimension $n$, and the Sobolev norms of the coefficients such that

$$
\|u\|_{L_{x}^{q}\left(\mathbb{R}^{n} ; L_{t}^{2}\left[-t_{0}, t_{0}\right]\right)} \leq C\left(\|f\|_{H^{\delta(q)}}+\|g\|_{H^{\delta(q)-1}}+\|F\|_{L_{t}^{1}\left(\left[-t_{0}, t_{0}\right] ; H^{\delta(q)-1}\left(\mathbb{R}^{n}\right)\right)}\right)
$$

for $q \geq q_{n}$ and any solution $u$ to the Cauchy problem (1.6).

Theorem 1.2. Assume that $\rho-1, a_{i j}-\delta_{i j} \in L^{r, \kappa}\left(\mathbb{R}^{n}\right) \cap \operatorname{Lip}\left(\mathbb{R}^{n}\right)$, with $\kappa=\frac{n-1}{r}+$ $1+\alpha$. Suppose also that $\sigma=\frac{1-\alpha}{3+\alpha}, q \geq q_{n}$ and $\delta(q)+\frac{\sigma}{q} \leq 1$. Then there exists $C$ depending only on the time interval, the dimension $n$, and the Sobolev/Lipschitz norms of the coefficients such that

$\|u\|_{L_{x}^{q}\left(\mathbb{R}^{n} ; L_{t}^{2}\left[-t_{0}, t_{0}\right]\right)} \leq C\left(\|f\|_{H^{\delta(q)+\frac{\sigma}{q}}}+\|g\|_{H^{\delta(q)+\frac{\sigma}{q}-1}}+\|F\|_{L^{1}\left(\left[-t_{0}, t_{0}\right] ; H^{\delta(q)+\frac{\sigma}{q}-1}\left(\mathbb{R}^{n}\right)\right)}\right)$ for any solution $u$ to the Cauchy problem (1.6).

Note that $\delta\left(q_{n}\right)+\frac{\sigma}{q_{n}} \leq 1$, yielding squarefunction estimates (1.2), (1.5) over compact manifolds for a range of indices that includes the critical index $q_{n}$. By the techniques of [10, this in turn proves spectral cluster estimates at the critical index for manifolds with metrics of the corresponding degree of Sobolev regularity. In 9, Smith used heat kernel methods to show that when $g \in \operatorname{Lip}(M)$, the spectral cluster estimate (1.1) holds for $q=\infty$ with no additional increase in the power of $\lambda$. As a consequence we can interpolate Smith's results with our spectral cluster estimates at the critical index to obtain the following:

Theorem 1.3. Let $M$ be a $C^{\infty}$ manifold equipped with a $L^{r, \kappa}(M) \cap \operatorname{Lip}(M)$ metric $g$ with $\kappa=\frac{n-1}{r}+1+\alpha$ where either $\alpha>1$ or $0<\alpha<1$. The spectral projector $\Pi_{\lambda}$ induced by $\Delta_{g}$ then satisfies, for $\sigma=\max \left(0, \frac{1-\alpha}{3+\alpha}\right)$,

$$
\begin{array}{lrl}
\left\|\Pi_{\lambda}\right\|_{L^{2}(M) \rightarrow L^{q}(M)} \leq C \lambda^{\frac{n-1}{2}\left(\frac{1}{2}-\frac{1}{q}\right)(1+\sigma)}, & 2 \leq q \leq \frac{2(n+1)}{n-1}, \\
\left\|\Pi_{\lambda}\right\|_{L^{2}(M) \rightarrow L^{q}(M)} \leq C \lambda^{n\left(\frac{1}{2}-\frac{1}{q}\right)-\frac{1}{2}+\frac{\sigma}{q}}, & \frac{2(n+1)}{n-1} \leq q \leq \infty .
\end{array}
$$

We remark that this theorem yields an improvement over what would be obtained by embedding the metric into a space $C^{s}$ and applying the current results in [9], 5]. This is because it allows us to control the operator norm of $\Pi_{\lambda}$ by a smaller power of $\lambda$ than would otherwise be needed.

In light of recent examples of Koch-Smith-Tataru [5], the assumption that $g$ is a Lipschitz metric is crucial for large values of $q$ in Theorem 1.3. In their work, for each $0<s<1, \lambda \gg 1$, they produce functions $\psi_{\lambda}, \rho_{\lambda}$ over $\mathbb{R}^{n}$ with $\rho_{\lambda}>0$, such that

$$
\Delta \psi_{\lambda}+\lambda^{2} \rho_{\lambda} \psi_{\lambda}=0, \quad \frac{\left\|\psi_{\lambda}\right\|_{L^{\infty}}}{\left\|\psi_{\lambda}\right\|_{L^{2}}} \approx \lambda^{\frac{n-s}{2}}, \quad \rho_{\lambda}=1+\lambda^{-s} q_{1}(\lambda|x|)+\lambda^{-2 s} q_{2}(\lambda|x|) .
$$


Here $\Delta$ is the standard Laplacian on Euclidean space and $q_{1}, q_{2}$ are smooth functions independent of $\lambda$. It is also seen that $\left\|\rho_{\lambda}-1\right\|_{C^{s}(M)}$ is uniformly bounded in $\lambda$. The $\psi_{\lambda}$ are exponentially concentrated to balls of radius $\lambda^{s-1}$ allowing one to lift a sequence of these examples to a compact manifold and produce a compact $(M, g)$ with $g \in C^{s}(M)$ a Hölder metric for which the best possible $L^{\infty}$ spectral cluster estimate is

$$
\left\|\Pi_{\lambda}\right\|_{L^{2}(M) \rightarrow L^{\infty}(M)} \leq C \lambda^{\frac{n-s}{2}} .
$$

Computing the $L^{r, \kappa}$ Sobolev norm of $\rho_{\lambda}-1$ yields

$$
\left\|\rho_{\lambda}-1\right\|_{L^{r, \kappa}}=\left\|\lambda^{-s} q_{1}(\lambda|\cdot|)+\lambda^{-2 s} q_{2}(\lambda|\cdot|)\right\|_{L^{r, \kappa}} \leq C \lambda^{\kappa-\frac{n}{r}-s} .
$$

Thus when $0<\kappa-\frac{n}{r}<1$ and $L^{r, \kappa}$ does not embed into a space of Lipschitz functions, setting $s=\kappa-\frac{n}{r}$ above also provides an example of an $L^{r, \kappa}$ metric for which the $L^{\infty}$ spectral cluster estimate $\left\|\Pi_{\lambda}\right\|_{L^{2}(M) \rightarrow L^{\infty}(M)} \leq C \lambda^{\frac{n-1}{2}}$ fails.

Using an argument of $\mathrm{H}$. Smith [9], we can also see that we must have

$$
\left\|\Pi_{\lambda}\right\|_{L^{2}(M) \rightarrow L^{q}(M)} \gtrsim \lambda^{\frac{n-s}{2}-\frac{n}{q}} .
$$

This uses the heat kernel methods alluded to above that were employed by Smith to show the $L^{2} \rightarrow L^{\infty}$ spectral cluster estimate for Lipschitz metrics. Consider $H_{\lambda}=\exp \left(-\lambda^{-2} \Delta_{g}\right)$, the heat evolution operator at time $\lambda^{-2}$. By Theorem 6.3 of Saloff-Coste 7 we have a pointwise bound over the integral kernel $h_{\lambda}$ of $H_{\lambda}$,

$$
\left|h_{\lambda}(x, y)\right| \leq C \lambda^{n} \exp \left(-c \lambda^{2} d_{g}(x, y)^{2}\right),
$$

with $d_{g}(x, y)$ denoting Riemannian distance. By Young's inequality we now have

$$
\lambda^{\frac{n-s}{2}} \leq C \frac{\left\|\psi_{\lambda}\right\|_{L^{\infty}}}{\left\|\psi_{\lambda}\right\|_{L^{2}}} \leq C^{\prime} \frac{\lambda^{\frac{n}{q}}\left\|H_{\lambda}^{-1} \psi_{\lambda}\right\|_{L^{q}}}{\left\|\psi_{\lambda}\right\|_{L^{2}}} \leq C^{\prime \prime} \lambda^{\frac{n}{q}} \exp \left(\frac{\lambda^{2}}{\lambda^{2}}\right) \frac{\left\|\psi_{\lambda}\right\|_{L^{q}}}{\left\|\psi_{\lambda}\right\|_{L^{2}}},
$$

which shows the claim. This now shows that (1.12) fails for a range of $q$ in an interval of the form $(c, \infty]$. For small $r$, values of $\alpha$ can be chosen so that $c<\frac{2(n+1)}{n-1}$, and hence (1.11) fails for certain $q$ as well. Hence the assumption that $g \in \operatorname{Lip}(M)$ is indeed crucial.

Unfortunately, our methods do not handle metrics in $L^{r, \kappa}$ when $\kappa=\frac{n-1}{r}+1+\alpha$ and $\alpha=0$ or 1 . In both cases, Sobolev embedding fails to provide the necessary control over the derivatives of the metric needed to prove the estimates. Indeed, if $\alpha=1$, (1.9) fails to hold for multi-indices $|\beta|=2$, precluding us from showing estimates of the form (1.2). When $\alpha=0$, (1.10) fails to hold, meaning that the truncation/rescaling methods here do not yield (1.5) with $\sigma=\frac{1}{3}$. In other words when $\alpha=0$ we are unable to provide any results, and when $\alpha=1$ the best we can provide is squarefunction estimates with an arbitrarily small loss of derivatives.

We also comment that the results of this paper yield homogeneous Strichartz estimates in dimensions $n \geq 4$. Indeed, the reductions in section 2 below are similar to those in [3], and the estimates (4.19), (5.14), (5.16) can be combined to establish the key dispersive estimates. However, we shall not pursue this issue in detail.

The paper is organized as follows: section 2 parallels the preliminaries in [3], reducing the problem to showing $L_{x}^{q} L_{t}^{2}$ estimates on solutions to the wave equation that are dyadically localized in frequency to regions contained in a set of the form (1.8). In section 3 , we then reduce the problem further, showing that it will suffice to find estimates on solutions to a first order equation that is hyperbolic in $x_{1}$ with coefficients satisfying (1.9). Section 4 then uses a continuous wave packet 
transform to construct a parametrix for the first order equation. It is then shown in section 5 that this parametrix yields an effective method for proving the desired estimates, culminating in a proof of Theorem 1.1 and Theorem 1.2

Notation. In what follows $d$ will denote the gradient operator which maps scalar functions to vector fields and vector fields to matrix functions in the natural way. When appropriate, subscripts will denote which variables are being differentiated. For example, $d_{x}$ will denote that spatial derivatives are taken and $d_{x, t}$ denotes the full space-time gradient.

Given a Lebesgue measurable rectangle $U \times V \subset \mathbb{R}^{n} \times \mathbb{R}, L_{x}^{q} L_{t}^{p}(U \times V)$ and $L_{x}^{q} L_{t}^{p}$ will abbreviate the Banach space $L^{q}\left(U ; L^{p}(V)\right)$, the latter being used when $U \times V=\mathbb{R}^{n+1}$. Similarly, $L_{t}^{p} H^{z}$ abbreviates $L^{p}\left(I ; H^{z}\right)$ when $I$ is an interval understood from the context of the argument.

The expression $X \lesssim Y$ means that $X \leq C Y$ for some $C$ depending only on $n$ and on the Lipschitz $/ L^{r, \kappa}$ norms of the coefficients. Additionally, $X \ll Y$ means that $X \leq C\left(c_{0}\right) Y$, where $C\left(c_{0}\right)$ denotes a constant depending on the choice of $c_{0}$ that can be made arbitrarily small by choosing $c_{0}$ small.

Lastly, $b_{j}(x)$ will always denote the function $b_{j}(x)=\sum_{i=1}^{n}\left(\partial_{i} a_{i j}\right)(x)$ so that we can write our wave operator in non-divergence form with a first order term

$$
P(x, D)=\rho(x) \partial_{t}^{2}-\sum_{1 \leq i, j \leq n} a_{i j}(x) \partial_{i} \partial_{j}-\sum_{j=1}^{n} b_{j}(x) \partial_{j} .
$$

\section{Preliminary Reductions}

We begin by laying out the first set of reductions in showing the squarefunction estimates. This is very similar to the approach used to show Strichartz estimates in [3. The main idea here is to reduce the problem to showing estimates on the components of $u$ frequency localized to dyadic regions contained in cones of the form $|\tau|>>\left|\left(\xi_{2}, \ldots, \xi_{n}\right)\right|$. Recall that we are interested in proving estimates over a finite time interval and that $q \geq q_{n}=\frac{2(n+1)}{n-1}$.

In this section, we assume that $a_{i j}, \rho \in L^{r, \kappa}\left(\mathbb{R}^{n}\right) \cap \operatorname{Lip}\left(\mathbb{R}^{n}\right)$ with $\kappa=\frac{n-1}{r}+1+\alpha$ and work with 2 sets of assumptions:

$$
\begin{aligned}
& \alpha>1, \quad \sigma=0, \\
& 0 \leq \delta(q) \leq \frac{3}{2}, \\
& 0<\alpha<1, \quad \sigma=\frac{1-\alpha}{3+\alpha}, \quad 0 \leq \delta(q)+\frac{\sigma}{q} \leq 1 .
\end{aligned}
$$

These correspond to the assumptions in Theorem 1.1 and Theorem 1.2, respectively. We now comment on the energy estimates that can be established for our wave operator.

Proposition 2.1. Suppose $t \in[-2,2]$. Solutions $u$ to the Cauchy problem (1.6) satisfy

$$
\begin{aligned}
& \|u(\cdot, t)\|_{H^{z}}+\left\|\partial_{t} u(\cdot, t)\right\|_{H^{z-1}} \\
& \quad \leq C\left(\|u(\cdot, 0)\|_{H^{z}}+\left\|\partial_{t} u(\cdot, 0)\right\|_{H^{z-1}}+\int_{0}^{t}\|F(\cdot, s)\|_{H^{z-1}} d s\right)
\end{aligned}
$$

with $0 \leq z \leq \frac{3}{2}$ when (2.1) is satisfied and $0 \leq z \leq 1$ when (2.2) is satisfied. 
Proof. We first show that (2.3) holds with $0 \leq z \leq 1$ under the weaker assumption that $a_{i j}, \rho$ are merely Lipschitz. First observe that the case $z=1$ follows from a standard computation. To handle the $z=0$ case when $\rho \equiv 1$, we observe that $\langle D\rangle^{-1} u$ (with $\langle D\rangle$ denoting the Fourier multiplier with symbol $\langle\xi\rangle=\left(1+|\xi|^{2}\right)^{\frac{1}{2}}$ ) satisfies the equation

$$
\begin{gathered}
P\left(\langle D\rangle^{-1} u\right)=\langle D\rangle^{-1} F-\sum_{1 \leq i, j \leq n} \partial_{x_{i}}\left[a_{i j},\langle D\rangle^{-1}\right] \partial_{x_{j}} u, \\
\langle D\rangle^{-1} u(x, 0)=\langle D\rangle^{-1} f, \quad \partial_{t}\langle D\rangle^{-1} u(x, 0)=\langle D\rangle^{-1} g .
\end{gathered}
$$

An application of the Coifman-Meyer commutator estimate (see (2.12) below) yields

$$
\left\|\partial_{x_{i}}\left[a_{i j},\langle D\rangle^{-1}\right] \partial_{x_{j}} u(\cdot, t)\right\|_{L^{2}} \lesssim\|u(\cdot, t)\|_{L^{2}}
$$

which in turn gives the desired energy estimate by Gronwall's inequality. To handle the $z=0$ case for variable $\rho(x)$ we observe that the function $w(x, t)=\rho(x) u(x, t)$ satisfies the equation

$$
\begin{gathered}
\partial_{t}^{2} w-\sum_{1 \leq i, j \leq n} \partial_{x_{j}}\left(a_{i j} \rho^{-1} \partial_{x_{i}} w\right)=F+\sum_{1 \leq i, j \leq n} \partial_{x_{j}}\left(a_{i j}\left(\partial_{x_{i}} \rho^{-1}\right) w\right), \\
w(x, 0)=\rho f, \quad \partial_{t} w(x, 0)=\rho g .
\end{gathered}
$$

We now apply the energy estimates above for $\rho \equiv 1, z=0$ to the wave operator on the left hand side of the first equation and use the fact that

$$
\left\|\partial_{x_{j}}\left(a_{i j}\left(\partial_{x_{i}} \rho^{-1}\right) w(\cdot, t)\right)\right\|_{H^{-1}} \lesssim\|w(\cdot, t)\|_{L^{2}}
$$

to get the complete energy estimates in the $z=0$ case. The full estimates for $0 \leq z \leq 1$ are a result of interpolation.

To establish inequalities when $1 \leq z \leq \frac{3}{2}$ we consider the equation satisfied by $\langle D\rangle^{\frac{1}{2}} u$ :

$$
\begin{aligned}
\rho \partial_{t}^{2}\left(\langle D\rangle^{\frac{1}{2}} u\right)-\sum_{1 \leq i, j \leq n} a_{i j} \partial_{i} \partial_{j}\left(\langle D\rangle^{\frac{1}{2}} u\right)-\sum_{j=1}^{n} b_{j} \partial_{j}\left(\langle D\rangle^{\frac{1}{2}} u\right) \\
=\langle D\rangle^{\frac{1}{2}} F+\sum_{j=1}^{n}\left[b_{j},\langle D\rangle^{\frac{1}{2}}\right] \partial_{j} u+\sum_{1 \leq i, j \leq n}\left[a_{i j},\langle D\rangle^{\frac{1}{2}}\right] \partial_{i} \partial_{j} u .
\end{aligned}
$$

Since $a_{i j} \in \operatorname{Lip}$ we can apply standard energy estimates with $z=1$ to the wave operator on the left hand side. To control the error terms on the right hand side we observe the following inequalities:

$$
\begin{gathered}
\left\|\left[a_{i j},\langle D\rangle^{\frac{1}{2}}\right] \partial_{i} \partial_{j}(u(\cdot, t))\right\|_{L^{2}} \lesssim\|u(\cdot, t)\|_{H^{\frac{3}{2}}}, \\
\left\|\left[b_{j},\langle D\rangle^{\frac{1}{2}}\right] \partial_{j}(u(\cdot, t))\right\|_{L^{2}} \lesssim\|u(\cdot, t)\|_{H^{\frac{3}{2}}}
\end{gathered}
$$

The first inequality now follows by the commutator estimate from (2.11). The second is a consequence of the fact that $b_{j} \in L^{r, \kappa-1}, \kappa-1>\frac{n}{r}+1-\frac{1}{r}$, implies that multiplication by $b_{j}$ is a bounded operation on $H^{\frac{1}{2}}$. Indeed, when $2<r<\infty$, $L^{r, \kappa-1} \hookrightarrow C^{\frac{1}{2}+\delta}$ for some $\delta>0$ and when $1<r \leq 2, L^{r, \kappa-1} \hookrightarrow L^{2, \frac{n}{2}+1-\frac{1}{r}}$.

Interpolation now proves the claim.

We make a few additional assumptions about our coefficients and our solution. Assume that $a_{i j}-\delta_{i j}, \rho-1 \in \operatorname{Lip}\left(\mathbb{R}^{n}\right)$ and are compactly supported with

$$
\left\|a_{i j}-\delta_{i j}\right\|_{L i p},\|\rho-1\|_{L i p}<c_{0}
$$


for some sufficiently small but fixed $c_{0}>0$. In addition, when $\kappa>\frac{n-1}{r}+2$ we assume that

$$
\sup _{T \in O(n)} \sup _{|\beta| \leq 2}\left\|\partial_{x}^{\beta}\left(a_{i j}(T \cdot)-\delta_{i j}\right)\right\|_{L_{x_{1}}^{r} L_{x^{\prime}}^{\infty}}<c_{0} .
$$

Lastly, we assume that $t_{0}=1$ and that $u(\cdot, t)$ is supported in the cube $\left(-\frac{3}{2}, \frac{3}{2}\right)^{n}$ for all $t \in\left(-\frac{5}{4}, \frac{5}{4}\right)$. The extra hypotheses are superfluous, as the general case can be reduced to these assumptions. This involves showing that we can restrict our attention to solutions supported in small sets and then rescaling the problem. See [16, pp. 394-395, for a suitable approach that also works in the context of squarefunction estimates as well as pp. 3-4 and p. 6 of [10].

We next observe that it suffices to prove the squarefunction estimates in Theorems 1.1 and 1.2 with $F \equiv 0$. By Duhamel's principle it is not difficult to see that the general case reduces to such an assumption. However, in order to be able to take the Fourier Transform of our solution, we make a small alteration of our solution so that it is compactly supported in time. We replace our solution to the homogeneous equation $u(x, t)$ by $\phi(t) u(x, t)$, where $\phi(t)$ is a smooth bump function equal to 1 on $[-1,1]$ and supported in $\left(-\frac{5}{4}, \frac{5}{4}\right)$.

This modification of $u$ means that it is no longer a solution to the homogeneous problem. However, energy estimates developed above allow us to control $[P, \phi]$ so that we have the following inequality for all $t \in \mathbb{R}$ provided that $0 \leq z \leq 1$ when $a_{i j} \in \operatorname{Lip}\left(\mathbb{R}^{n}\right)$ or that $0 \leq z \leq \frac{3}{2}$ when $a_{i j}-\delta_{i j} \in L^{r, \kappa}\left(\mathbb{R}^{n}\right), \kappa>\frac{n-1}{r}+2$ :

$$
\|u(\cdot, t)\|_{H^{z}}+\left\|\partial_{t} u(\cdot, t)\right\|_{H^{z-1}}+\|(P u)(\cdot, t)\|_{H^{z-1}} \lesssim\|f\|_{H^{z}}+\|g\|_{H^{z-1}} .
$$

We have now defined $u(x, t)$ as a function over all of $\mathbb{R}^{n+1}$, so it now makes sense to look at $u$ as an element of a Sobolev space in $n+1$ variables. Hence $H_{x, t}^{z}$ will denote the $L^{2}$ Sobolev space of order $z$ on $\mathbb{R}^{n+1}$ in the $(x, t)$ variables and $H^{z}$ will denote the Sobolev space on $\mathbb{R}^{n}$ in only the $x$ variables.

To localize in frequency we first take a sequence of Littlewood-Paley cutoffs $\left\{\beta_{k}\right\}_{k \geq 0}$, so that $\beta_{k}(\xi)$ is a smooth function for $\xi \in \mathbb{R}^{n}$ with $\sum_{k} \beta_{k} \equiv 1, \operatorname{supp}\left(\beta_{k}\right) \subset$ $\left\{\xi \in \mathbb{R}^{n}: 2^{k-\frac{1}{2}} \leq|\xi| \leq 2^{k+\frac{3}{2}}\right\}$ when $k \neq 0$, and $\operatorname{supp}\left(\beta_{0}\right) \subset B_{2}(0)$. We can also take the sequence so that $\beta_{k}(\cdot)=\beta\left(2^{-k} \cdot\right)$ for $k>1$ for some function $\beta \in C_{0}^{\infty}\left(\mathbb{R}^{n}\right)$.

By multiplying $\beta_{0}$ by another smooth cutoff in $\tau$, we can extend $\beta_{0}$ to be a smooth cutoff supported in a ball of radius 2 in $\mathbb{R}^{n+1}$ and one in a neighborhood of the origin in $\mathbb{R}^{n+1}$; that is, there exists $\bar{\beta}_{0}(\xi, \tau)$ with the above properties such that $\bar{\beta}_{0}(\xi, 0)=\beta_{0}(\xi)$. Treating $\bar{\beta}_{0}(D)$ as a Fourier multiplier on $u$ in the $(x, t)$ variables, $\bar{\beta}_{0}(D)$ is a smoothing operator. Hence by Sobolev embedding and energy estimates we obtain the following for coefficients satisfying (2.1) or (2.2):

$$
\left\|\bar{\beta}_{0}(D) u\right\|_{L_{x}^{q} L_{t}^{2}\left([-1,1] \times \mathbb{R}^{n}\right)} \lesssim\|u\|_{L_{t}^{2} H^{\delta(q)+\frac{\sigma}{q}}} \lesssim\|f\|_{H^{\delta(q)+\frac{\sigma}{q}}}+\|g\|_{H^{\delta(q)+\frac{\sigma}{q}-1}} .
$$

Now take a cutoff $\Lambda^{+} \in C^{\infty}\left(\mathbb{R}^{n+1} \backslash\{0\}\right)$ homogeneous of degree 0 such that $\operatorname{supp}\left(\Lambda^{+}\right) \subset\left\{(\xi, \tau): 9^{-1}|\xi| \leq \tau \leq 9|\xi|\right\}$ and is identically 1 on the slightly smaller cone $\left\{(\xi, \tau): 8^{-1}|\xi| \leq \tau \leq 8|\xi|\right\}$. Since we already have estimates on $\bar{\beta}_{0} u$ we will replace $\Lambda^{+}$by $\Lambda^{+}\left(I-\bar{\beta}_{0}\right)$ so that $\Lambda^{+}$is smooth on all of $\mathbb{R}^{n+1}$ and vanishes in a neighborhood of the origin. Also, set $\Lambda^{-}(\xi, \tau)=\Lambda^{+}(\xi,-\tau)$. We now need to control the $L_{x}^{q} L_{t}^{2}$ norm of the following:

$$
\Lambda^{+}(D) u, \Lambda^{-}(D) u \text {, and }\left(I-\Lambda^{+}(D)-\Lambda^{-}(D)-\bar{\beta}_{0}(D)\right) u .
$$

The latter component can be estimated using elliptic regularity. 
Proposition 2.2. Suppose that $a_{i j}, \rho \in \operatorname{Lip}\left(\mathbb{R}^{n}\right)$. Then we have the following estimate whenever $0 \leq \delta(q) \leq \frac{3}{2}$ :

$$
\left\|\left(I-\Lambda^{+}(D)-\Lambda^{-}(D)-\bar{\beta}_{0}(D)\right) u\right\|_{L_{x}^{q} L_{t}^{2}\left(\mathbb{R}^{n+1}\right)} \lesssim\|f\|_{H^{\delta(q)}}+\|g\|_{H^{\delta(q)-1}} .
$$

Proof. The proof is inspired by Lemma 2.3 of [10]. For $k \geq 1$, set

$$
\alpha_{k}(\xi, \tau)=\left(I-\Lambda^{+}(\xi, \tau)-\Lambda^{-}(\xi, \tau)\right) \beta_{k}(\xi) .
$$

We let $\tilde{\alpha}_{k}(\xi, \tau)$ be a multiplier such that $\tilde{\alpha}_{k}(\xi, \tau) \alpha_{k}(\xi, \tau)=\alpha_{k}(\xi, \tau)$, and be supported in only a slightly larger set than $\operatorname{supp}\left(\alpha_{k}\right)$ (in particular, supported away from the characteristic set of $P$ ).

For each $k$, introduce a second decomposition, writing $\alpha_{k}(\xi, \tau)=\sum_{k=j}^{\infty} \alpha_{k, j}(\xi, \tau)$, where

$$
\operatorname{supp}\left(\alpha_{k, j}\right) \subset\left\{\begin{array}{l}
|\tau| \approx 2^{j},|\xi| \approx 2^{k}, \quad k \gg j, \\
|\tau| \lesssim 2^{k},|\xi| \approx 2^{k}, \quad k \approx j .
\end{array}\right.
$$

Again, we define $\tilde{\alpha}_{k, j}(\xi, \tau)$ to be a multiplier such that $\tilde{\alpha}_{k, j}(\cdot) \alpha_{k, j}(\cdot)=\alpha_{k, j}(\cdot)$, and with slightly larger support. In particular, we may assume that $\operatorname{supp}\left(\tilde{\alpha}_{k, j}\right)$ is contained in a set of the form (2.6) (with different implied constants).

By Minkowski's inequality, Littlewood-Paley theory and (2.5), it suffices to show that

$$
\sum_{k=1}^{\infty} \sum_{j=k}^{\infty}\left\|\alpha_{k, j}(D) u\right\|_{L_{t}^{2} L_{x}^{q}}^{2} \lesssim\|u\|_{L_{t}^{2} H^{\delta(q)}}^{2}+\left\|\partial_{t} u\right\|_{L_{t}^{2} H^{\delta(q)-1}}^{2}+\|P u\|_{L_{t}^{2} H^{\delta(q)-1}}^{2} .
$$

First observe that by Sobolev embedding (or Young's inequality applied to the integral kernel of $\tilde{\alpha}_{k, j}$ ) we have

$$
\left\|\alpha_{k, j} u\right\|_{L_{t}^{2} L_{x}^{q}} \lesssim 2^{k\left(\delta(q)+\frac{1}{2}\right)}\left\|\alpha_{k, j} u\right\|_{L_{x, t}^{2}} .
$$

Hence we need elliptic regularity to counter this loss of half a derivative. We define

$$
a_{i j}^{k}=\sum_{l \leq \frac{k}{2}} \beta_{l}(D) a_{i j}, \quad \rho^{k}=\sum_{l \leq \frac{k}{2}} \beta_{l}(D) \rho, \quad \text { and } \quad P^{k}(\cdot, D)=\rho^{k} \partial_{t}^{2}-\sum_{i j} \partial_{i}\left(a_{i j}^{k} \partial_{j}\right) .
$$

Observe that since $a_{i j}(x) \in \operatorname{Lip}\left(\mathbb{R}^{n}\right)$ we always have the crude estimates

$$
\left\|a_{i j}^{k}-a_{i j}\right\|_{L^{\infty}} \lesssim 2^{-\frac{k}{2}}, \quad\left\|d_{x} a_{i j}^{k}\right\|_{L^{\infty}} \lesssim 1
$$

and similar estimates for $\rho^{k}$. By choosing $c_{0}$ sufficiently small in (2.4),$P^{k}$ is also a perturbation of the constant coefficient wave operator. Thus $P^{k}(x, D) \circ \tilde{\alpha}_{k, j}(D)$ is an elliptic operator with symbol estimates in $S_{1, \frac{1}{2}}^{2}$ uniformly bounded in $k$. In addition, the first derivatives of the symbol in $x$ are also in $S_{1, \frac{1}{2}}^{2}$. Hence there exist symbols

$$
q^{k, j}(x, \xi, \tau) \in S_{1, \frac{1}{2}}^{-2}\left(\mathbb{R}^{2 n+2}\right), \quad \quad r^{k, j}(x, \xi, \tau) \in S_{1, \frac{1}{2}}^{-\frac{1}{2}}\left(\mathbb{R}^{2 n+2}\right)
$$

with

$$
I=\sum_{j \geq k} q^{k, j}(x, D) \circ P^{k}(x, D) \circ \tilde{\alpha}_{k, j}(D)+r^{k}(x, D),
$$

$I$ denoting the identity operator. Recall that the regularized coefficients are truncated in frequency to a set $\left\{|\xi| \lesssim 2^{k / 2}\right\}$. Hence the Fourier transform of the 
$P^{k}(x, D)\left(\alpha_{k, j}(D) u\right)$ is supported in only a slightly larger set than the $\alpha_{k, j}(D) u$. This means there is no harm in assuming that symbol $q^{k, j}$ is supported in a set

$$
\operatorname{supp}\left(q^{k, j}(x, \cdot)\right) \subset\left\{\begin{array}{l}
|\tau| \approx 2^{j},|\xi| \approx 2^{k}, \quad k \gg j, \\
|\tau| \lesssim 2^{k},|\xi| \approx 2^{k}, \quad k \approx j .
\end{array}\right.
$$

This implies that the integral kernel of $q^{k, j}(x, D)$ is pointwise bounded by

$$
C_{N} 2^{-2 j} 2^{j+k n}\left(1+2^{j}|t-s|+2^{k}|x-y|\right)^{-N}
$$

for any $N>0$, allowing us to conclude that

$$
2^{\frac{k}{2}}\left\|\alpha_{k, j}(D) u\right\|_{L_{x, t}^{2}} \lesssim 2^{-2 j+\frac{k}{2}}\left\|P^{k}(x, D)\left(\alpha_{k, j}(D) u\right)\right\|_{L_{x, t}^{2}}+\left\|\alpha_{k, j}(D) u\right\|_{L_{x, t}^{2}} .
$$

Now write

$$
P^{k}\left(\alpha_{k, j}(D) u\right)=\left(P^{k}-P\right) \alpha_{k, j}(D) u+\alpha_{k, j}(D) P u+\left[P, \alpha_{k, j}(D)\right] u .
$$

Using the estimates (2.8) on $\rho-\rho^{k}, a_{i j}-a_{i j}^{k}$ we have that

$$
\begin{aligned}
2^{-2 j+\frac{k}{2}}\left\|\left(P^{k}-P\right) \alpha_{k, j}(D) u\right\|_{L_{x, t}^{2}} & \lesssim 2^{-2 j}\left(\left\|d_{x, t}^{2} \alpha_{k, j}(D) u\right\|_{L_{x, t}^{2}}+\left\|d_{x, t} \alpha_{k, j}(D) u\right\|_{L_{x, t}^{2}}\right) \\
& \lesssim\left\|\alpha_{k, j}(D) u\right\|_{L_{x, t}^{2}} .
\end{aligned}
$$

It is thus straightforward to control all terms not involving a commutator. Indeed, by almost orthogonality of the $\alpha_{k, j}$, the sum

$$
\sum_{j \geq k} 2^{2 k\left(\delta(q)+\frac{1}{2}\right)-4 j}\left\|P^{k}(x, D) \alpha_{k, j}(D) u-\left[P, \alpha_{k, j}(D)\right] u\right\|_{L_{x, t}^{2}}^{2}+\sum_{j \geq k}\left\|\alpha_{k, j}(D) u\right\|_{L_{x, t}^{2}}^{2}
$$

is bounded by the right hand side of (2.7). Hence it suffices to show that

$$
\sum_{k \geq j} 2^{2 k\left(\delta(q)+\frac{1}{2}\right)-4 j}\left\|\left[P, \alpha_{k, j}(D)\right] u\right\|_{L_{x, t}^{2}}^{2} \lesssim\left\|\partial_{t} u\right\|_{L_{t}^{2} H^{\delta(q)-1}}^{2}+\|u\|_{L_{t}^{2} H^{\delta(q)}}^{2} .
$$

Let $m=m(k, j)$ be an enumeration of the set $\left\{(k, j) \in \mathbb{N}^{2}: k \geq j\right\}$. The key idea here will be to apply Lemma 2.4 of 3 which implies that if $R_{\theta}=$ $\sum_{k \geq j} e^{i m \theta} 2^{k\left(\delta(q)+\frac{1}{2}\right)-2 j} \alpha_{k, j}$ and

$$
\left\|\left[P, R_{\theta}\right] u\right\|_{L_{x, t}^{2}} \lesssim\left\|\partial_{t} u\right\|_{L_{t}^{2} H^{\delta(q)-1}}+\|u\|_{L_{t}^{2} H^{\delta(q)}},
$$

then (2.9) holds.

In order to obtain (2.10), we use an important corollary of the Coifman-Meyer commutator theorem. Suppose $R$ is a Fourier multiplier of order $z$ and $a$ is a Lipschitz function. Then the commutator of $R$ with the multiplication operation $f \mapsto a \cdot f$ enjoys the following continuous mapping properties:

$$
\begin{array}{ll}
{[a, R]: H^{z-1}\left(\mathbb{R}^{n+1}\right) \rightarrow L^{2}\left(\mathbb{R}^{n+1}\right),} & 0 \leq z \leq 1, \\
{[a, R]: H^{z}\left(\mathbb{R}^{n+1}\right) \rightarrow H^{1}\left(\mathbb{R}^{n+1}\right),} & -1 \leq z \leq 0 .
\end{array}
$$

The Coifman-Meyer result handles the $z=1$ case, and the $z=0,-1$ cases then follow easily. Interpolation then provides the result for the intermediate values of z. See Proposition 3.6B and the surrounding discussion in Section 3.6 of [19] for a more detailed discussion of this result. 
Returning to our operator $R_{\theta}$, observe that it is a Fourier multiplier of order $\delta(q)-\frac{3}{2}$ with symbol estimates uniform in $\theta$. We can exploit the fact that $P$ is a divergence form operator to obtain (2.10)

$$
\begin{aligned}
\left\|\left[P, R_{\theta}\right] u\right\|_{L_{x, t}^{2}} & \lesssim\left\|\left[\rho, R_{\theta}\right] \partial_{t} u\right\|_{H_{x, t}^{1}}+\sum_{i j}\left\|\left[a_{i j}, R_{\theta}\right] \partial_{j} u\right\|_{H_{x, t}^{1}} \\
& \lesssim\left\|d_{x, t} u\right\|_{H_{x, t}^{\max \left(-1, \delta(q)-\frac{3}{2}\right)}} \lesssim\|u\|_{L_{t}^{2} H^{\delta(q)}}+\left\|\partial_{t} u\right\|_{L_{t}^{2} H^{\delta(q)-1}}
\end{aligned}
$$

The squarefunction estimates for $\Lambda^{+} u, \Lambda^{-} u$ will require further localization. Let $\Phi(\xi) \in C^{\infty}\left(\mathbb{R}^{n} \backslash\{0\}\right)$ be a cutoff such that

$$
\operatorname{supp}(\Phi) \subset\left\{\xi:-\xi_{1}>M\left|\xi^{\prime}\right|\right\}
$$

and identically 1 on a slightly smaller cone, where $M$ is a sufficiently large constant to be determined. Set $\Gamma^{ \pm}(\xi, \tau)=\Phi(\xi) \Lambda^{ \pm}(\xi, \tau)$. By employing a family of similar cutoffs, we can represent $\Lambda^{ \pm} u$ as a finite sum of functions supported in cones in $\xi$ of comparable aperture, so it will suffice to show estimates for the localized $\Gamma^{ \pm}(D) u$. Also, we will show them for $\Gamma^{-} u$, as estimates for $\Gamma^{+} u$ will follow by an identical argument; so from now on we will suppress the ' - ' in $\Gamma^{-}$and refer to it simply as $\Gamma$.

Set $\Gamma_{k}(\xi, \tau)=\beta_{k}(\xi) \Gamma(\xi, \tau)$. Also, let $\psi$ be a smooth cutoff in $x_{1}$ that is identically 1 on a neighborhood of $\left[-\frac{3}{2}, \frac{3}{2}\right]$ and supported in $(-2,2)$ so that $\psi\left(x_{1}\right) u(x, t)=$ $u(x, t)$. We pause to mention that for any $x_{1} \in \mathbb{R}$, the partial Fourier transform of $\psi \Gamma_{k} u$ in $x^{\prime}, t$ is supported in the set

$$
\operatorname{supp}\left(\widehat{\psi \Gamma_{k} u}\right)\left(x_{1}, \cdot\right) \subset\left\{\left(\xi^{\prime}, \tau\right):-\tau \geq \widetilde{M}\left|\xi^{\prime}\right|\right\} \cap\left\{\left(\xi^{\prime}, \tau\right): 2^{k-5} \leq\left|\left(\xi^{\prime}, \tau\right)\right| \leq 2^{k+6}\right\}
$$

where $\widetilde{M}$ is a constant depending on $M$ in (2.14) that can be made arbitrarily large by choosing $M$ sufficiently large.

The key result of this section will be to show that the inequalities on $\Gamma u$ reduce to estimates on the $\psi \Gamma_{k} u$ :

Proposition 2.3. Suppose $\psi \Gamma_{k} u$ satisfies the following estimates uniformly in $k$ whenever (2.1) or (2.2) hold:

$$
\begin{array}{r}
\left\|\psi \Gamma_{k} u\right\|_{L_{x}^{q} L_{t}^{2}\left([-2,2] \times \mathbb{R}^{n}\right)} \lesssim 2^{k\left(\delta(q)+\frac{\sigma}{q}\right)}\left(\left\|\psi \Gamma_{k} u\right\|_{L_{x_{1}}^{\infty} L_{x^{\prime}, t}^{2}}+2^{-k}\left\|d_{x, t}\left(\psi \Gamma_{k} u\right)\right\|_{L_{x_{1}}^{\infty} L_{x^{\prime}, t}^{2}}\right. \\
+\left\|\Gamma_{k} u\right\|_{L_{x, t}^{2}}+2^{-k}\left\|P\left(\psi \Gamma_{k} u\right)\right\|_{\left.L_{x_{1} L_{x^{\prime}, t}^{1}}^{2}\right) .} .
\end{array}
$$

Then $u$ satisfies the corresponding squarefunction estimates in Theorem 1.1 or Theorem 1.2 with $F \equiv 0$.

Note that $L_{x}^{q} L_{t}^{2}\left([-2,2] \times \mathbb{R}^{n}\right)$ abbreviates $L_{x_{1}, x^{\prime}}^{q}\left([-2,2] \times \mathbb{R}^{n-1} ; L_{t}^{2}(\mathbb{R})\right)$.

Proof. The proof here is very similar to that in Theorem 2.1 in 3]. As in Proposition 2.2, it suffices to show

$$
\|\Gamma u\|_{L_{x}^{q} L_{t}^{2}} \lesssim\|u\|_{L_{t}^{2} H^{\delta(q)+\frac{\sigma}{q}}}+\left\|\partial_{t} u\right\|_{L_{t}^{2} H^{\delta(q)+\frac{\sigma}{q}-1}}+\|P u\|_{L_{t}^{2} H^{\delta(q)+\frac{\sigma}{q}-1}} .
$$

We first observe that the projections of the $\Gamma_{k}(\xi, \tau)$ onto the $\tau$ axis have bounded overlap, meaning that Littlewood-Paley theory implies

$$
\|\Gamma u\|_{L_{x}^{q} L_{t}^{2}} \approx\left\|\Gamma_{k} u\right\|_{L_{x}^{q} L_{t}^{2} l_{k}^{2}} \leq\left\|\psi \Gamma_{k} u\right\|_{l_{k}^{2} L_{x}^{q} L_{t}^{2}}+\left\|(1-\psi) \Gamma_{k} u\right\|_{l_{k}^{2} L_{x}^{q} L_{t}^{2}}
$$


Since $\Gamma_{k} u$ is given by convolution of $u$ against a Schwartz function concentrated in a ball of radius $2^{-k}$ about the origin, we have that for any $N>0$

$$
\left\|(1-\psi) \Gamma_{k} u\right\|_{L_{x}^{q} L_{t}^{2}} \leq C_{N} 2^{-k N}\|u\|_{L_{x, t}^{2}},
$$

with $C_{N}$ independent of $k$. This implies that

$$
\left\|(1-\psi) \Gamma_{k} u\right\|_{l_{k}^{2} L_{x}^{q} L_{t}^{2}} \lesssim\|u\|_{L_{t}^{2} H^{\delta(q)+\frac{\sigma}{q}}} .
$$

Hence it will suffice to control the square sum over the $\psi \Gamma_{k} u$.

We now need to control the $L_{x_{1}}^{\infty} L_{x^{\prime}, t}^{2}$ norm of $d_{x, t}\left(\psi \Gamma_{k} u\right)$. For this we apply the flux estimates in Proposition 2.2 of [3] which imply that for any $r_{1}, y_{1} \in[-2,2]$ we have that

$$
\left\|d_{x, t}\left(\psi \Gamma_{k} u\right)\left(y_{1}, \cdot\right)\right\|_{L_{x^{\prime}, t}^{2}} \lesssim\left\|d_{x, t}\left(\psi \Gamma_{k} u\right)\left(r_{1}, \cdot\right)\right\|_{L_{x^{\prime}, t}^{2}}+\int_{r_{1}}^{y_{1}}\left\|P\left(\psi \Gamma_{k} u\right)\left(z_{1}, \cdot\right)\right\|_{L_{x^{\prime}, t}^{2}} d z_{1} .
$$

The key idea here is that since the $\psi \Gamma_{k} u$ are localized to a region where $P$ behaves as an operator that is hyperbolic in $x_{1}$, we can formulate energy estimates in the $x_{1}$ variable for such functions. Since $2^{k}\left\|\psi \Gamma_{k} u\right\|_{L_{x_{1}}^{\infty} L_{x^{\prime}, t}^{2}} \lesssim\left\|\partial_{t} \psi \Gamma_{k} u\right\|_{L_{x_{1}}^{\infty} L_{x^{\prime}, t}^{2}}$, we can choose $r_{1}=-2$ in this inequality to obtain

$$
2^{k}\left\|\psi \Gamma_{k} u\right\|_{L_{x_{1}}^{\infty} L_{x^{\prime}, t}^{2}}+\left\|d_{x, t}\left(\psi \Gamma_{k} u\right)\right\|_{L_{x_{1}}^{\infty} L_{x^{\prime}, t}^{2}} \lesssim\left\|P\left(\psi \Gamma_{k} u\right)\right\|_{L_{x_{1}}^{1} L_{x^{\prime}, t}^{2}} .
$$

Hence (2.15) implies that

$$
\left\|\psi \Gamma_{k} u\right\|_{L_{x}^{q} L_{t}^{2}\left([-2,2] \times \mathbb{R}^{n}\right)} \lesssim 2^{k\left(\delta(q)+\frac{\sigma}{q}-1\right)}\left(2^{k}\left\|\Gamma_{k} u\right\|_{L_{x, t}^{2}}+\left\|P\left(\psi \Gamma_{k} u\right)\right\|_{L_{x_{1}}^{1} L_{x^{\prime}, t}^{2}}\right) .
$$

We now write

$$
P\left(\psi \Gamma_{k} u\right)=[P, \psi] \Gamma_{k} u+\psi\left[P, \Gamma_{k}\right] u+\psi \Gamma_{k} P u .
$$

Since the inequality

$$
\begin{aligned}
\sum_{k} 2^{2 k\left(\delta(q)+\frac{\sigma}{q}-1\right)}\left(\left\|\psi \Gamma_{k} P u\right\|_{L_{x_{1}}^{1} L_{x^{\prime}, t}^{2}}^{2}\right. & \left.+\left\|[P, \psi] \Gamma_{k} u\right\|_{L_{x, t}^{2}}^{2}+2^{2 k}\left\|\Gamma_{k} u\right\|_{L_{x, t}^{2}}^{2}\right) \\
& \lesssim\|P u\|_{L_{t}^{2} H^{\delta(q)+\frac{\sigma}{q}-1}}^{2}+\|u\|_{L_{t}^{2} H^{\delta(q)+\frac{\sigma}{q}}}^{2}
\end{aligned}
$$

is straightforward, the key step in the proof will lie in showing that

$$
\begin{aligned}
\sum_{k} 2^{2 k\left(\delta(q)+\frac{\sigma}{q}-1\right)}\left\|\psi\left[P, \Gamma_{k}\right] u\right\|_{L_{x_{1}}^{1} L_{x^{\prime}, t}^{2}}^{2} & \\
& \lesssim\|u\|_{L_{t}^{2} H^{\delta(q)}+\frac{\sigma}{q}}^{2}+\left\|\partial_{t} u\right\|_{L_{t}^{2} H^{\delta(q)+\frac{\sigma}{q}-1}}^{2}+\|P u\|_{L_{t}^{2} H^{\delta(q)+\frac{\sigma}{q}-1}}^{2} .
\end{aligned}
$$

This is where the proof of our statement varies from that of Theorem 2.1 in 3 .

We now employ an application of Lemma 2.4 in [3] similar to that used in Proposition 2.2, this time setting $R_{\theta}=\sum_{k=0}^{\infty} e^{i k \theta} 2^{k\left(\delta(q)+\frac{\sigma}{q}-1\right)} \Gamma_{k}$. In the case where $0 \leq \delta(q)+\frac{\sigma}{q} \leq 1$, the corollary of the Coifman-Meyer theorem stated above together with an argument similar to that in (2.13) imply that

$$
\left\|\psi\left[P, R_{\theta}\right] u\right\|_{L_{x, t}^{2}} \lesssim\|u\|_{L_{t}^{2} H^{\delta(q)+\frac{\sigma}{q}}}+\left\|\partial_{t} u\right\|_{L_{t}^{2} H^{\delta(q)+\frac{\sigma}{q}-1}},
$$

and hence (2.16) follows as before.

The case $1 \leq \delta(q)+\frac{\sigma}{q} \leq \frac{3}{2}$ is only considered when $\sigma=0$ and $\kappa>\frac{n-1}{r}+2$, as we will need the additional regularity of the coefficients in this instance. Since 
$\sigma=0$, we drop it in the remainder of the proof. Once again, it suffices to establish mapping properties of $\left[P, R_{\theta}\right]$. In this case, we write

$$
\left[P, R_{\theta}\right]=\left[\rho, R_{\theta}\right] \partial_{t}^{2}-\sum_{i j}\left[a_{i j}, R_{\theta}\right] \partial_{x_{i} x_{j}}^{2}-\sum_{j}\left[b_{j}, R_{\theta}\right] \partial_{x_{j}} .
$$

We now use (2.11) to get that

$$
\left[a_{i j}, R_{\theta}\right]: H^{\delta(q)-2}\left(\mathbb{R}^{n+1}\right) \rightarrow L^{2}\left(\mathbb{R}^{n+1}\right)
$$

with operator norm independent of $\theta$. This in turn implies that

$$
\left\|\psi\left[P+\sum_{j=1}^{n} b_{j} \partial_{x_{j}}, R_{\theta}\right] u\right\|_{L_{x, t}^{2}}^{2} \lesssim \sum_{l=0}^{2}\left\|\partial_{t}^{l} u\right\|_{L_{t}^{2} H^{\delta(q)-l}} .
$$

To control the sum on the right, observe that since $\frac{1}{\rho(x)} \in \operatorname{Lip}\left(\mathbb{R}^{n}\right)$, multiplication by this function is a bounded operation on $H^{\delta(q)-2}\left(\mathbb{R}^{n}\right)$, and hence the equation gives us that

$$
\left\|\partial_{t}^{2} u\right\|_{L_{t}^{2} H^{\delta(q)-2}} \lesssim\left\|\rho \partial_{t}^{2} u\right\|_{L_{t}^{2} H^{\delta(q)-2}} \lesssim\|P u\|_{L_{t}^{2} H^{\delta(q)-2}}+\|A(\cdot, D) u\|_{L_{t}^{2} H^{\delta(q)-2}} .
$$

Since $A(x, D)$ is a divergence form operator with Lipschitz coefficients, we have that $A(x, D): H^{\delta(q)} \rightarrow H^{\delta(q)-2}$ and hence

$$
\sum_{l=0}^{2}\left\|\partial_{t}^{l} u\right\|_{L_{t}^{2} H^{\delta(q)-l}} \lesssim\|u\|_{L_{t}^{2} H^{\delta(q)}}+\left\|\partial_{t} u\right\|_{L_{t}^{2} H^{\delta(q)-1}}+\|P u\|_{L_{t}^{2} H^{\delta(q)-1}} .
$$

Turning our attention to the first order terms, observe that the higher degree of regularity in the coefficients implies that multiplication by the function $b_{j}$ is a bounded operation on $H^{\delta(q)-1}\left(\mathbb{R}^{n}\right)$. This follows by essentially the same considerations in Proposition 2.1. When $1<r \leq 2$, Sobolev embedding tells us that $\partial_{i} a_{i j} \in H^{\frac{n}{2}+1-\frac{1}{r}}$, and when $2<r<\infty, \partial_{i} a_{i j} \in C^{1-\frac{1}{r}}$. In either case, this implies that

$$
\left\|\left[b_{j}, R_{\theta}\right] \partial_{x_{j}} u\right\|_{L^{2}} \lesssim\left\|\partial_{x_{j}} u\right\|_{L_{t}^{2} H^{\delta(q)-1}}
$$

and hence $\left[P, R_{\theta}\right]$ possesses the desired mapping properties.

\section{THE HALF-WAVE OPERATOR}

In this section, we show that the $\psi \Gamma_{k} u$ are also solutions to a first-order pseudodifferential equation that is hyperbolic in $x_{1}$. In future sections, we will construct a parametrix for this first-order operator that yields the desired squarefunction estimates. Throughout this discussion, we assume that $a_{i j}-\delta_{i j} \in L^{r, \kappa}$ with $\kappa=\frac{n-1}{r}+1+\alpha, 1<r<\infty$, and either $\alpha>1$ or $0<\alpha<1$.

To construct the first order pseudodifferential operator, we let $\Psi \in C^{\infty}\left(\mathbb{R}^{n}\right)$ be a cutoff function in $\left(\xi^{\prime}, \tau\right)$ such that $\operatorname{supp}(\Psi) \subset\left\{\left(\xi^{\prime}, \tau\right):-\tau \geq 5\left|\xi^{\prime}\right|\right\}$ and is identically 1 on the smaller region $\left\{\left(\xi^{\prime}, \tau\right):-\tau \geq 6\left|\xi^{\prime}\right|,\left|\left(\xi^{\prime}, \tau\right)\right| \geq \frac{1}{2}\right\}$. We can also take the function so that

$$
\Psi\left(\xi^{\prime}, \tau\right)=\Psi\left(\left(\xi^{\prime}, \tau\right) /\left|\left(\xi^{\prime}, \tau\right)\right|\right) \quad \text { for }\left|\left(\xi^{\prime}, \tau\right)\right| \geq \frac{3}{4} .
$$

Let $\tilde{a}_{i j}=a_{11}^{-1} a_{i j}, \tilde{\rho}_{i j}=a_{11}^{-1} \rho_{i j}, \tilde{b}_{j}=a_{11}^{-1} \sum_{i} \partial_{i} a_{i j}$, and set

$$
\tilde{a}_{i j}^{k}=\sum_{l \leq \varsigma k} \beta_{l}(D) \tilde{a}_{i j}
$$


where $\varsigma=\max \left(\frac{1}{2}, \frac{2}{3+\alpha}\right)$. Define $Q$ as the pseudodifferential operator with rough symbol,

$$
\begin{aligned}
Q(x, \xi, \tau)=\xi_{1}^{2} & +\left(2 \sum_{j=2}^{n} \tilde{a}_{1 j}(x) \xi_{1} \xi_{j}-\left(\tilde{\rho}(x) \tau^{2}-\sum_{2 \leq i, j \leq n} \tilde{a}_{i j}(x) \xi_{i} \xi_{j}\right)\right) \Psi\left(\xi^{\prime}, \tau\right) \\
& -\left(\tau^{2}+\sum_{i=2}^{n} \xi_{i}^{2}\right)\left(1-\Psi\left(\xi^{\prime}, \tau\right)\right)
\end{aligned}
$$

and let $Q_{k}$ be the analogous operator defined by replacing the $\tilde{a}_{i j}$ in the symbol defining $Q$ with their regularized counterparts $\tilde{a}_{i j}^{k}$. Observe that by construction, if $w(x, t)$ is a function such that $\operatorname{supp}(\widehat{w}) \subset \Psi^{-1}(1)$, then

$$
Q w=\frac{1}{a_{11}} P w+\sum_{i j} \frac{\partial_{i} a_{i j}}{a_{11}} \partial_{j} w .
$$

In particular, by choosing $M \geq 200$ in (2.14), this holds for the $\psi \Gamma_{k} u$. On the other hand, since $Q(x, \xi, \tau)$ and $Q_{k}(x, \xi, \tau)$ are quadratics in $\xi_{1}$ with real roots, these operators are hyperbolic in $x_{1}$ rather than in $t$.

We now let $\varrho\left(x_{1}\right)$ be the Hardy-Littlewood maximal function associated to the $L^{r}(\mathbb{R})$ function

$$
\sum_{|\beta| \leq \min (\alpha, 1)+1} \sum_{i, j}\left\|\partial_{x}^{\beta}\left(a_{i j}\left(x_{1}, \cdot\right)-\delta_{i j}\right)\right\|_{L_{x^{\prime}}^{\infty}}+\sum_{|\beta| \leq \min (\alpha, 1)+1}\left\|\partial_{x}^{\beta}\left(\rho\left(x_{1}, \cdot\right)-1\right)\right\|_{L_{x^{\prime}}^{\infty} .}
$$

Note that since $1<r<\infty, \varrho\left(x_{1}\right) \in L^{r}(\mathbb{R})$ as well. We now claim that

$$
\begin{aligned}
\left\|\left(\tilde{a}_{i j}-\tilde{a}_{i j}^{k}\right)\left(x_{1}, \cdot\right)\right\|_{L_{x^{\prime}}^{\infty}} & \leq C 2^{-k \min (1, \varsigma(1+\alpha))} \varrho\left(x_{1}\right), \\
\left\|\partial_{x}^{\beta} \tilde{a}_{i j}^{k}\left(x_{1}, \cdot\right)\right\|_{L_{x^{\prime}}^{\infty}} & \leq C_{\beta} 2^{k \varsigma \max (0,|\beta|-1-\alpha)} \varrho\left(x_{1}\right), \\
\left\|\partial_{x}^{\beta} \tilde{a}_{i j}^{k}\right\|_{L^{\infty}} & \leq C_{\beta} 2^{k \varsigma \max (0,|\beta|-1)} .
\end{aligned}
$$

Since $a_{i j} \in$ Lip, the estimate (3.3) follows by a straightforward argument. The estimates (3.1), (3.2) follow by essentially the same arguments as in section 3 and section 5 of [3]. If $a \in L^{r, \kappa}$, set $\beta_{l}(D) a=\beta_{l}(D)\langle D\rangle^{-\kappa}\left(\langle D\rangle^{\kappa} a\right)$. Hence if $\tilde{\phi}_{l}=$ $\mathcal{F}^{-1}\left\{\beta_{l}(\xi)\langle\xi\rangle^{-\kappa}\right\}$, we then have $\beta_{l}(D) a=\left(\langle D\rangle^{\kappa} a\right) * \tilde{\phi}_{l}$ and the following estimates on $\tilde{\phi}_{l}$ :

$$
\left|y^{\beta_{1}} \partial_{y}^{\beta_{2}} \tilde{\phi}_{l}(y)\right| \leq C_{\beta_{1}, \beta_{2}} 2^{l\left(n-\left|\beta_{1}\right|+\left|\beta_{2}\right|-\kappa\right)}, \quad\left\|\partial_{y}^{\beta} \tilde{\phi}_{l}(y)\right\|_{L^{r^{\prime}}} \leq C_{\beta} 2^{l(|\beta|-1-\alpha)} .
$$

An application of standard results on maximal functions (see Theorem 2 in Chapter III, Section 2 of Stein [14]) now yields

$$
\left|\partial_{x}^{\beta}\left(\beta_{l}(D) a\right)(x)\right|=\left|\left(\langle D\rangle^{\kappa} a\right) *\left(\partial_{x}^{\beta} \tilde{\phi}_{l}\right)(x)\right| \leq C_{\beta} 2^{l(|\beta|-1-\alpha)} \varrho\left(x_{1}\right) .
$$

Summing this estimate with $|\beta|=0$ over all $l>k \varsigma$ now yields (3.1). Similarly, (3.2) follows by summing this estimate over all $l \leq k \varsigma$ for any $\beta$.

We now decompose

$$
Q_{k}(x, \xi, \tau)=\left(\xi_{1}+q_{k}^{-}\left(x, \xi^{\prime}, \tau\right)\right)\left(\xi_{1}-q_{k}^{+}\left(x, \xi^{\prime}, \tau\right)\right)
$$

so that $q_{k}^{ \pm}\left(x, \xi^{\prime}, \tau\right)$ are positive functions that yield the roots of $Q_{k}(x, \xi, \tau)$ as a quadratic in $\xi_{1}$. Next set

$$
p_{k}\left(x, \xi^{\prime}, \tau\right)=\sum_{l \leq \varsigma k} \beta_{l}\left(D_{x}\right) q_{k}^{-}\left(x, \xi^{\prime}, \tau\right)
$$


so that when $|\eta| \geq 2^{k \varsigma}$,

$$
\int e^{-i\langle x, \eta\rangle} p_{k}\left(x, \xi^{\prime}, \tau\right)=0 .
$$

In addition, if $\hat{w}_{k}\left(\xi^{\prime}, \tau\right)$ is a function supported in a set $K$, then the Fourier transform of $p_{k}\left(x, D_{x^{\prime}, t}\right) w_{k}$ will be supported in a set of the form $K+\left\{\left|\left(\xi^{\prime}, \tau\right)\right| \leq C 2^{k \varsigma}\right\}$. Note that similar considerations to those used to establish (3.1) now yield

$$
\sup _{\left|\left(\xi^{\prime}, \tau\right)\right|=1}\left|\partial_{x}^{\gamma} \partial_{\xi^{\prime}, \tau}^{\beta}\left(p_{k}\left(x, \xi^{\prime}, \tau\right)-q_{k}^{-}\left(x, \xi^{\prime}, \tau\right)\right)\right| \leq C_{\gamma, \beta} \varrho\left(x_{1}\right) 2^{-k \min (1, \varsigma(1+\alpha))+k \varsigma|\gamma|}
$$

for perhaps some slightly different $\varrho \in L^{r}$. Also, if $\Xi=\left\{\left(\xi^{\prime}, \tau\right) \in \mathbb{S}^{n-1}:-\tau \geq 6\left|\xi^{\prime}\right|\right\}$,

$$
\sup _{\left(\xi^{\prime}, \tau\right) \in \Xi}\left|\partial_{\xi^{\prime}, \tau}^{\beta}\left(p_{k}\left(x, \xi^{\prime}, \tau\right)-\sqrt{\tau^{2}-\left|\xi^{\prime}\right|^{2}}\right)\right| \leq C_{\gamma, \beta} \cdot c_{0},
$$

which follows from the fact that $q_{k}^{-}$satisfies a similar estimate by (2.4). In addition, we observe that by the same arguments used to estimate $a_{i j}^{k}(\cdot), q_{k}^{-}\left(\cdot, \xi^{\prime}, \tau\right)$, $p_{k}\left(\cdot, \xi^{\prime}, \tau\right)$ satisfy the same bounds (3.2) and (3.3) uniformly in $\left(\xi^{\prime}, \tau\right)$. Lastly, we will only need the fact that $\rho \in L_{l o c}^{r} \subset L_{l o c}^{1}$, so there is no harm in assuming that

$$
\rho\left(x_{1}\right) \gtrsim 1
$$

pointwise by adding a constant if necessary.

For the rest of this section we will take the convention that $\sigma=\max \left(0, \frac{1-\alpha}{3+\alpha}\right)$. By the arguments in section 5 of [3], there exists a collection of $\mathcal{O}\left(2^{k \sigma}\right)$ subintervals, $\left\{I_{m}^{k}\right\}_{m}$, such that $\bigcup_{m} I_{m}^{k}=(-2,2)$, and for each $m$,

$$
\left|I_{m}^{k}\right| \leq 2^{-k \sigma}, \quad \int_{I_{m}^{k}} \varrho\left(x_{1}\right) d x_{1} \ll 2^{-k \sigma} .
$$

This follows by taking a maximal collection of subintervals of $(-2,2)$ satisfying (3.6). By maximality of the collection it can be seen that there must be $\mathcal{O}\left(2^{k \sigma}\right)$ such subintervals. See 3 for more details.

We now want to rescale the problem. Set $w_{k}(x, t)=\psi \Gamma_{k} u\left(2^{-k \sigma}(x, t)\right), \lambda=$ $2^{k(1-\sigma)}$, and $\tilde{p}_{\lambda}\left(x, \xi^{\prime}, \tau\right)=p_{k}\left(2^{-k \sigma} x, \xi^{\prime}, \tau\right)$. As a result, $\widehat{w}_{k}(\xi, \tau)$ is localized to frequencies $\approx \lambda$. Also, since $\varsigma-\sigma=\frac{1}{2}(1-\sigma)$,

$$
\int e^{-i\langle x, \eta\rangle} \tilde{p}_{\lambda}\left(x, \xi^{\prime}, \tau\right) d x=0 \text { for }|\eta| \geq \lambda^{\frac{1}{2}} .
$$

We also set $\tilde{\varrho}\left(x_{1}\right)=\varrho\left(2^{-k \sigma} x_{1}\right)$ so that by (ㅎ․6), $\|\tilde{\varrho}\|_{L^{r}\left(2^{k \sigma} I_{m}^{k}\right)} \ll 1$ over the set

$$
2^{k \sigma} I_{m}^{k}=\left\{2^{k \sigma} x_{1}: x_{1} \in I_{m}^{k}\right\}
$$

Rescaling the estimates in (3.2) and (3.3) (which as observed also apply to $\left.p_{k}\left(\cdot, \xi^{\prime}, \tau\right)\right)$, we have the following estimates on the partial derivatives of $\tilde{p}_{\lambda}$ :

$$
\begin{aligned}
& \sup _{\left(x, \xi^{\prime}, \tau\right) \in \mathbb{R}^{n} \times \mathbb{S}^{n-1}}\left|\partial_{x}^{\alpha} \partial_{\xi}^{\beta} \tilde{p}_{\lambda}\left(x, \xi^{\prime}, \tau\right)\right| \leq C_{\alpha, \beta} \lambda^{\frac{1}{2}(\max (0,|\alpha|-1))}, \\
& \sup _{\left(x^{\prime}, \xi^{\prime}, \tau\right) \in \mathbb{R}^{n-1} \times \mathbb{S}^{n-1}}\left|\partial_{x}^{\alpha} \partial_{\xi}^{\beta} \tilde{p}_{\lambda}\left(x, \xi^{\prime}, \tau\right)\right| \leq C_{\alpha, \beta} \tilde{\varrho}\left(x_{1}\right) \lambda^{\frac{1}{2}(\max (0,|\alpha|-2))},
\end{aligned}
$$


with $C_{\alpha, \beta}$ independent of $\lambda$. Combining this with (3.5), we have that

$$
\begin{aligned}
& \sup _{x \in \mathbb{R}^{n},\left(\xi^{\prime}, \tau\right) \in \Xi} \sum_{|\alpha|+|\beta| \leq 2,|\alpha| \leq 1}\left|\partial_{x}^{\alpha} \partial_{\xi}^{\beta}\left(\tilde{p}_{\lambda}\left(x, \xi^{\prime}, \tau\right)-\sqrt{\tau^{2}-\left|\xi^{\prime}\right|^{2}}\right)\right| \ll 1, \\
& \sup _{x^{\prime} \in \mathbb{R}^{n-1},\left(\xi^{\prime}, \tau\right) \in \Xi} \sum_{|\alpha|+|\beta| \leq 2}\left|\partial_{x}^{\alpha} \partial_{\xi}^{\beta}\left(\tilde{p}_{\lambda}\left(x, \xi^{\prime}, \tau\right)-\sqrt{\tau^{2}-\left|\xi^{\prime}\right|^{2}}\right)\right| \leq \tilde{\varrho}\left(x_{1}\right),
\end{aligned}
$$

where again $\Xi=\left\{\left(\xi^{\prime}, \tau\right) \in \mathbb{S}^{n-1}:-\tau \geq 6\left|\xi^{\prime}\right|\right\}$.

In the next two sections, we prove the following theorem:

Theorem 3.1. Let $I \subset[-2,2]$ be any interval containing 0 and $p\left(x, \xi^{\prime}, \tau\right)$ a symbol satisfying (3.7), (3.9), (3.10), (3.11), and (3.12). Suppose $v$ solves the first order equation

$$
\begin{aligned}
\left(\partial_{x_{1}}+i p\left(x, D_{x^{\prime}, t}\right)\right) v(x, t) & =F(x, t) \in L_{x_{1}}^{1}\left(I ; L_{x^{\prime}, t}^{2}\left(\mathbb{R}^{n}\right)\right), \\
v\left(0, x^{\prime}, t\right) & =v_{0}\left(x^{\prime}, t\right) \in L^{2}\left(\mathbb{R}^{n}\right)
\end{aligned}
$$

and that for any $x_{1} \in I$

$$
\begin{aligned}
\operatorname{supp}\left(\widehat{v}\left(x_{1}, \cdot\right)\right) & \operatorname{supp}\left(\widehat{F}\left(x_{1}, \cdot\right)\right) \\
& \subset\left\{\left(\xi^{\prime}, \tau\right): 2^{-5} \lambda \leq\left|\left(\xi^{\prime}, \tau\right)\right| \leq 2^{6} \lambda\right\} \cap\left\{\left(\xi^{\prime}, \tau\right):-\tau \geq \widetilde{M}\left|\xi^{\prime}\right|\right\}
\end{aligned}
$$

for some $\lambda, \widetilde{M}$ sufficiently large. Then

$$
\|v\|_{L_{x_{1}, x^{\prime}}^{q}\left(I \times \mathbb{R}^{n-1} ; L_{t}^{2}(\mathbb{R})\right)} \lesssim \lambda^{\delta(q)}\left(\left\|v_{0}\right\|_{L^{2}}+\|F\|_{L_{x_{1}}^{1}\left(I ; L_{x^{\prime}, t}^{2}\left(\mathbb{R}^{n}\right)\right)}\right) .
$$

This theorem will imply the following estimate on $w_{k}$ over the set (3.8):

$$
\begin{aligned}
\left\|w_{k}\right\|_{L_{x}^{q} L_{t}^{2}\left(2^{k \sigma} I_{m}^{k} \times \mathbb{R}^{n}\right)} \lesssim 2^{k(1-\sigma) \delta(q)}\left(\left\|w_{k}\right\|_{L_{x_{1}}^{\infty} L_{x^{\prime}, t}^{2}\left(2^{k \sigma} I_{m}^{k} \times \mathbb{R}^{n}\right)}\right. \\
\left.+\left\|\left(-i \partial_{1}+\tilde{p}\left(x, D_{x^{\prime}, t}\right)\right) w_{k}\right\|_{L_{x_{1}}^{1} L_{x^{\prime}, t}^{2}\left(2^{k \sigma} I_{m}^{k} \times \mathbb{R}^{n}\right)}\right) .
\end{aligned}
$$

We can then rescale the estimate to obtain

$$
\begin{aligned}
\left\|\psi \Gamma_{k} u\right\|_{L_{x}^{q} L_{t}^{2}\left(I_{m}^{k} \times \mathbb{R}^{n}\right)} \lesssim 2^{k \delta(q)}\left(\left\|\psi \Gamma_{k} u\right\|_{L_{x_{1}}^{\infty} L_{x^{\prime}, t}^{2}\left(I_{m}^{k} \times \mathbb{R}^{n}\right)}\right. \\
+ \\
\left.+\left\|\left(-i \partial_{1}+p_{k}\left(\cdot, D_{x^{\prime}, t}\right)\right) \psi \Gamma_{k} u\right\|_{L_{x_{1}}^{1} L_{x^{\prime}, t}^{2}\left(I_{m}^{k} \times \mathbb{R}^{n}\right)}\right) .
\end{aligned}
$$

In order to see that this implies the estimates in the hypothesis of Proposition 2.3 , we will show following estimate:

$$
\begin{aligned}
\|\left(-i \partial_{1}+\right. & \left.p_{k}\left(\cdot, D_{x^{\prime}, t}\right)\right) \psi \Gamma_{k} u\left\|_{L_{x_{1}}^{1} L_{x^{\prime}, t}^{2}\left(I_{m}^{k} \times \mathbb{R}^{n}\right)} \lesssim\right\| \psi \Gamma_{k} u \|_{L_{x_{1}}^{\infty} L_{x^{\prime}, t}^{2}} \\
+ & 2^{-k}\left\|\partial_{1} \psi \Gamma_{k} u\right\|_{L_{x_{1}}^{\infty} L_{x^{\prime}, t}^{2}}+2^{-k}\left\|P\left(\psi \Gamma_{k} u\right)\right\|_{L_{x_{1}}^{1} L_{x^{\prime}, t}^{2}}^{2}+\left\|\Gamma_{k} u\right\|_{L_{x, t}^{2}}^{2} .
\end{aligned}
$$

Combining this with (3.14), we can sum over $m$ to obtain

$$
\begin{aligned}
& \left\|\psi \Gamma_{k} u\right\|_{L_{x}^{q} L_{t}^{2}} \lesssim\left(\sum_{m}\left\|\psi \Gamma_{k} u\right\|_{L_{x}^{q} L_{t}^{2}\left(I_{m}^{k} \times \mathbb{R}^{n}\right)}^{q}\right)^{\frac{1}{q}} \\
& \lesssim 2^{k\left(\delta(q)+\frac{\sigma}{q}\right)}\left(\left\|\psi \Gamma_{k} u\right\|_{L_{x_{1}}^{\infty} L_{x^{\prime}, t}^{2}}+2^{-k}\left\|\partial_{1} \psi \Gamma_{k} u\right\|_{L_{x_{1}}^{\infty} L_{x^{\prime}, t}^{2}}\right. \\
& \left.+2^{-k}\left\|P\left(\psi \Gamma_{k} u\right)\right\|_{L_{x_{1}}^{1} L_{x^{\prime}, t}^{2}}+\left\|\Gamma_{k} u\right\|_{L_{x, t}^{2}}\right) .
\end{aligned}
$$


Proof of (3.15). Let $\widetilde{\Gamma}_{1}(\tau, \xi)$ be a bump function that is identically one on the support of $\Gamma_{1}$ and is supported in a slightly larger cone containing $\operatorname{supp}\left(\Gamma_{1}\right)$. With this, define $\widetilde{\Gamma}_{k}(\cdot)=\widetilde{\Gamma}_{1}\left(2^{-k} \cdot\right)$ and choose the level set $\widetilde{\Gamma}_{1}^{-1}(1)$ large enough so that $\widetilde{\Gamma}_{k} \cdot \Gamma_{k}=\Gamma_{k}$ and

$$
\widetilde{\Gamma}_{k}(D) \circ\left(-i \partial_{1}-p_{k}\left(\cdot, D_{x^{\prime}, t}\right)\right) \Gamma_{k}(D)=\left(-i \partial_{1}-p_{k}\left(\cdot, D_{x^{\prime}, t}\right)\right) \Gamma_{k}(D) .
$$

We can now write

$$
\begin{aligned}
& \left(-i \partial_{1}+p_{k}\left(\cdot, D_{x^{\prime}, t}\right)\right) \psi \Gamma_{k} u \\
& \quad=\widetilde{\Gamma}_{k}\left(-i \partial_{1}+p_{k}\left(\cdot, D_{x^{\prime}, t}\right)\right) \psi \Gamma_{k} u-\left[\widetilde{\Gamma}_{k},\left(-i \partial_{1}+p_{k}\left(\cdot, D_{x^{\prime}, t}\right)\right) \psi\right] \Gamma_{k} u .
\end{aligned}
$$

By the symbolic calculus and (3.3), the commutator lies in $S_{1, \varsigma}^{0}\left(\mathbb{R}^{2 n+2}\right)$, and as such the $L^{2}$ norm of the second term can be bounded by the last term on the right hand side of (3.15). Rewrite the operator in the first term as

$$
\begin{aligned}
& \widetilde{\Gamma}_{k}\left(-i \partial_{1}+p_{k}\left(\cdot, D_{x^{\prime}, t}\right)\right)=\widetilde{\Gamma}_{k}\left(-i \partial_{1}+q_{k}^{-}\left(\cdot, D_{x^{\prime}, t}\right)\right) \\
& \quad+\left[\widetilde{\Gamma}_{k},\left(p_{k}\left(\cdot, D_{x^{\prime}, t}\right)-q_{k}^{-}\left(\cdot, D_{x^{\prime}, t}\right)\right)\right]+\left(p_{k}\left(\cdot, D_{x^{\prime}, t}\right)-q_{k}^{-}\left(\cdot, D_{x^{\prime}, t}\right)\right) \widetilde{\Gamma}_{k} .
\end{aligned}
$$

As above, the commutator term lies in $S_{1, \varsigma}^{0}\left(\mathbb{R}^{2 n+2}\right)$. By (3.4) and the fact that $1-\varsigma(1+\alpha)=\sigma$ when $\sigma>0$, we have that for $\left|\left(\xi^{\prime}, \tau\right)\right| \approx 2^{k}$

$$
\left|\partial_{x}^{\gamma} \partial_{\xi^{\prime}, \tau}^{\beta}\left(q_{k}^{-}\left(x, \xi^{\prime}, \tau\right)-p_{k}\left(x, \xi^{\prime}, \tau\right)\right)\right| \leq C_{\alpha, \beta} \varrho\left(x_{1}\right) 2^{k \sigma+k \varsigma|\gamma|-k|\beta|} .
$$

By (3.6),,$\|\varrho\|_{L^{1}\left(I_{m}^{k}\right)} \leq 2^{-k \sigma}$, which implies

$$
\left\|\left(q_{k}^{+}\left(\cdot, D_{x^{\prime}, t}\right)-p_{k}^{+}\left(\cdot, D_{x^{\prime}, t}\right)\right) \widetilde{\Gamma}_{k} \psi \Gamma_{k} u\right\|_{L_{x_{1}}^{1} L_{x^{\prime}, t}^{2}\left(I_{m}^{k} \times \mathbb{R}^{n}\right)} \lesssim\left\|\psi \Gamma_{k} u\right\|_{L_{x_{1}}^{\infty} L_{x^{\prime}, t}^{2}\left(I_{m}^{k} \times \mathbb{R}^{n}\right)} .
$$

To handle the first term in (3.16), let

$$
B_{k}(x, \xi, \tau)=\frac{\widetilde{\Gamma}_{k}(\xi, \tau)}{\xi_{1}-q_{k}^{+}\left(x, \xi^{\prime}, \tau\right)}, \quad \text { so that } \quad B_{k}, d_{x} B_{k} \in S_{1, \varsigma}^{-1} .
$$

These are well defined, as $\widetilde{\Gamma}_{k}$ is supported away from the zero set of the denominator. Integration by parts yields the following estimates on the integral kernel of $B_{k}$ :

$$
2^{k}\left|\partial_{x, t}^{\alpha} K_{k}(x, t ; y, s)\right| \lesssim 2^{k(n+1)}\left(1+2^{k}|(x, t)-(y, s)|\right)^{-(n+2)}, \quad|\alpha| \leq 1 .
$$

Observe that by (3.3) and the symbolic calculus we have

$$
\begin{gathered}
\widetilde{\Gamma}_{k}(D)=B_{k}(x, D) \circ\left(-i \partial_{1}-q_{k}^{+}(x, D)\right) \bmod S_{1, \varsigma}^{-1}, \\
\left(-i \partial_{1}-q_{k}^{+}\left(x, D_{x^{\prime}, t}\right)\right) \circ\left(-i \partial_{1}+q_{k}^{-}\left(x, D_{x^{\prime}, t}\right)\right)=Q_{k}(x, D) \bmod S_{1, \varsigma}^{1} .
\end{gathered}
$$

This allows us to write

$$
\widetilde{\Gamma}_{k}\left(-i \partial_{1}+q_{k}^{-}\left(\cdot, D_{x^{\prime}, t}\right)\right) \psi \Gamma_{k} u=\left(B_{k}(x, D) \circ Q_{k}(x, D)+R_{k}(x, D)\right) \psi \Gamma_{k} u
$$

where $R_{k}(x, \xi, \tau) \in S_{1, \varsigma}^{0}$. We have now shown that

$$
\begin{aligned}
& \left\|\left(-i \partial_{1}+p_{k}\left(\cdot, D_{x^{\prime}, t}\right)\right) \psi \Gamma_{k} u\right\|_{L_{x_{1}}^{1} L_{x^{\prime}, t}^{2}\left(I_{m}^{k} \times \mathbb{R}^{n}\right)} \\
& \quad \lesssim\left\|\psi \Gamma_{k} u\right\|_{L_{x_{1}}^{\infty} L_{x^{\prime}, t}^{2}\left(I_{m}^{k} \times \mathbb{R}^{n}\right)}+\left\|\Gamma_{k} u\right\|_{L_{x, t}^{2}}^{2}+\left\|B_{k} \circ Q_{k}\left(\psi \Gamma_{k} u\right)\right\|_{L_{x_{1}}^{1} L_{x^{\prime}, t}^{2}\left(I_{m}^{k} \times \mathbb{R}^{n}\right)} .
\end{aligned}
$$

To estimate the last term on the right hand side we write

$$
B_{k} \circ Q_{k}=\left[B_{k}, Q_{k}-Q\right]+\left(Q_{k}-Q\right) B_{k}+B_{k} Q .
$$


Using (3.18), we can directly estimate the kernel of $\left[B_{k}, a\right]$ for Lipschitz functions $a$ and use Schur's Lemma to get

$$
\left\|\left[B_{k}, Q_{k}-Q\right]\left(\psi \Gamma_{k} u\right)\right\|_{L_{x_{1}}^{1} L_{x^{\prime}, t}^{2}\left(I_{m}^{k} \times \mathbb{R}^{n}\right)} \lesssim 2^{-2 k}\left\|d_{x, t}^{2}\left(\psi \Gamma_{k} u\right)\right\|_{L_{x, t}^{2}} \lesssim\left\|\Gamma_{k} u\right\|_{L_{x, t}^{2}} .
$$

As in (3.17) we also have

$$
\left\|\left(Q_{k}-Q\right) B_{k}\left(\psi \Gamma_{k} u\right)\right\|_{L_{x_{1}}^{1} L_{x^{\prime}, t}^{2}\left(I_{m}^{k} \times \mathbb{R}^{n}\right)} \lesssim\left\|d_{x^{\prime}, t} B_{k}\left(\psi \Gamma_{k} u\right)\right\|_{L_{x_{1}}^{\infty} L_{x^{\prime}, t}^{2}} \lesssim\left\|\psi \Gamma_{k} u\right\|_{L_{x_{1}}^{\infty} L_{x^{\prime}, t}^{2}}
$$

after an application of (3.18) and Schur's Lemma in the second inequality. We now use (3.18) again to complete the proof by arguing that since $\rho>0$,

$$
\left\|B_{k} Q\left(\psi \Gamma_{k} u\right)\right\|_{L_{x_{1}}^{1} L_{x^{\prime}, t}^{2}\left(I_{m}^{k} \times \mathbb{R}^{n}\right)} \lesssim 2^{-k}\left\|P\left(\psi \Gamma_{k} u\right)\right\|_{L_{x_{1}}^{1} L_{x^{\prime}, t}^{2}}+2^{-k}\left\|\partial_{1}\left(\psi \Gamma_{k} u\right)\right\|_{L_{x_{1}}^{\infty} L_{x^{\prime}, t}^{2}} .
$$

\section{The WAVE TRANSFORM}

We have now reduced the problem of showing squarefunction inequalities for general solutions of the wave equation to estimating the $L_{x}^{q} L_{t}^{2}$ norm of solutions to a $x_{1}$-hyperbolic pseudodifferential equation. To show Theorem 3.1, we proceed by constructing a parametrix for such operators that yields the estimates. As previously mentioned, we employ the continuous wave packet transform used by Smith in 10] for this purpose.

For ease of exposition in the arguments we will reverse the roles of $t$ and $x_{1}$ and assume that our first order pseudodifferential equation is hyperbolic in $t$. Therefore, in this discussion and in section [5, we assume that $p(x, t, \xi)$ is a first order symbol close to $\sqrt{\xi_{1}^{2}-\left|\xi^{\prime}\right|^{2}}$, meaning that (3.11), (3.12), (3.10), and (3.9) take the form

$$
\begin{gathered}
\sup _{x \in \mathbb{R}^{n}, \xi \in \Xi} \sum_{|\alpha|+|\beta| \leq 2,|\alpha| \leq 1}\left|\partial_{x, t}^{\alpha} \partial_{\xi}^{\beta}\left(p(x, t, \xi)-\sqrt{\xi_{1}^{2}-\left|\xi^{\prime}\right|^{2}}\right)\right| \ll 1, \\
\sup _{x \in \mathbb{R}^{n}, \xi \in \Xi} \sum_{|\alpha|+|\beta| \leq 2}\left|\partial_{x, t}^{\alpha} \partial_{\xi}^{\beta}\left(p(x, t, \xi)-\sqrt{\xi_{1}^{2}-\left|\xi^{\prime}\right|^{2}}\right)\right| \leq \varrho(t), \\
\sup _{\left(x^{\prime}, \xi\right) \in \mathbb{R}^{n-1} \times \mathbb{S}^{n-1}}\left|\partial_{x, t}^{\alpha} \partial_{\xi}^{\beta} p(x, t, \xi)\right| \leq C_{\alpha, \beta} \lambda^{\frac{1}{2}(\max (0,|\alpha|-1))}, \\
\sup _{\left(x^{\prime}, \xi\right) \in \mathbb{R}^{n-1} \times \mathbb{S}^{n-1}}\left|\partial_{x, t}^{\alpha} \partial_{\xi}^{\beta} p(x, t, \xi)\right| \leq C_{\alpha, \beta} \varrho(t) \lambda^{\frac{1}{2}(\max (0,|\alpha|-2)),},
\end{gathered}
$$

where $\Xi=\left\{\xi \in \mathbb{S}^{n-1}:-\xi_{1} \geq 6\left|\xi^{\prime}\right|\right\}$ and again $\|\varrho\|_{L^{r}(I)} \ll 1$. We thus seek to show that solutions $v(x, t)$ to

$$
\begin{aligned}
\left(\partial_{t}+i p\left(x, t, D_{x}\right)\right) v(x, t) & =F(x, t) \in L_{t}^{1}\left(I ; L_{x}^{2}\left(\mathbb{R}^{n}\right)\right), \\
v(x, 0) & =v_{0}(x) \in L^{2}(\mathbb{R}),
\end{aligned}
$$

such that

$$
\operatorname{supp}(\widehat{v}(\cdot, t)), \operatorname{supp}(\widehat{F}(\cdot, t)) \subset\left\{\xi: 2^{-5} \lambda \leq|\xi| \leq 2^{6} \lambda\right\} \cap\left\{\xi:-\xi_{1} \geq \widetilde{M}\left|\xi^{\prime}\right|\right\},
$$

satisfy over a time interval $t \in[-2,2]$,

$$
\|v\|_{L_{x^{\prime}, t}^{q}\left(\mathbb{R}^{n-1} \times[-2,2] ; L_{x_{1}}^{2}(\mathbb{R})\right)} \lesssim \lambda^{\delta(q)}\left(\left\|v_{0}\right\|_{L^{2}}+\|F\|_{L_{t}^{1} L_{x}^{2}}\right) .
$$


We now introduce the continuous wave packet transform. Let $g$ be a radial Schwartz function over $\mathbb{R}^{n}$ such that $\operatorname{supp}(\widehat{g}) \subset B_{1}(0)$ and $\|g\|_{L^{2}}=(2 \pi)^{-\frac{n}{2}}$. For $\lambda \geq 1$, we define the operator $T_{\lambda}: \mathcal{S}^{\prime}\left(\mathbb{R}^{n}\right) \rightarrow C^{\infty}\left(\mathbb{R}^{2 n}\right)$ by

$$
\left(T_{\lambda} f\right)(x, \xi)=\lambda^{\frac{n}{4}} \int e^{-i\langle\xi, z-x\rangle} g\left(\lambda^{\frac{1}{2}}(z-x)\right) f(z) d z .
$$

$T_{\lambda}$ enjoys the property that its adjoint as a map from $L_{x, \xi}^{2}\left(\mathbb{R}^{2 n}\right) \rightarrow L_{z}^{2}\left(\mathbb{R}^{n}\right)$ also serves as a left inverse for $T_{\lambda}$; that is, $T_{\lambda}^{*} T_{\lambda}=I$. This implies that $T_{\lambda}$ is an isometry:

$$
\left\|T_{\lambda} f\right\|_{L_{x, \xi}^{2}\left(\mathbb{R}^{2 n}\right)}=\|f\|_{L_{z}^{2}\left(\mathbb{R}^{n}\right)} .
$$

Since $\widehat{g}(\eta)$ is supported in the unit ball, we have also that for any $x$,

$$
\operatorname{supp}\left(T_{\lambda} f(x, \cdot)\right) \subset \operatorname{supp}(\widehat{f})+2 \lambda^{\frac{1}{2}} .
$$

We will want to show (4.6) by considering $T_{\lambda}(v(\cdot, t))$. For each $t$, we conjugate $P\left(x, t, D_{x}\right)$ by $T_{\lambda}$ in $x$ and take a suitable approximation to the resulting operator. Define the following first order differential operator over $(x, \xi)$,

$$
\begin{aligned}
\widetilde{P(t)} & =i d_{\xi} p(x, t, \xi) \cdot d_{x}-i d_{x} p(x, t, \xi) \cdot d_{\xi}-p(x, t, \xi)+\xi \cdot d_{\xi} p(x, t, \xi) \\
& =i d_{\xi} p(x, t, \xi) \cdot d_{x}-i d_{x} p(x, t, \xi) \cdot d_{\xi}
\end{aligned}
$$

with the latter equality following by homogeneity. In Lemmas 3.1-3.3 of [10], H. Smith proved that if $\widetilde{\beta}_{\lambda}$ is a Littlewood-Paley cutoff truncating to frequencies $|\xi| \approx \lambda$, then

$$
\left\|T_{\lambda} p(\cdot, t, D) \widetilde{\beta}_{\lambda}(D)-\widetilde{P(t)} T_{\lambda} \widetilde{\beta}_{\lambda}(D)\right\|_{L_{z}^{2} \rightarrow L_{x, \xi}^{2}} \lesssim \varrho(t) .
$$

Setting $\tilde{v}(x, t, \xi)=T_{\lambda}(v(\cdot, t))(x, \xi), \tilde{F}(x, t, \xi)=T_{\lambda}(v(\cdot, t))(x, \xi)$, this implies the existence of a function $G(x, t, \xi)$ satisfying the equation

$$
\partial_{t} \tilde{v}=d_{\xi} p(x, t, \xi) \cdot d_{x} \tilde{v}-d_{x} p(x, t, \xi) \cdot d_{\xi} \tilde{v}+\tilde{F}+G
$$

as well as the estimate

$$
\|G(\cdot, t, \cdot)\|_{L_{x, \xi}^{2}\left(\mathbb{R}^{2 n}\right)} \lesssim \varrho(t)\|v(\cdot, t)\|_{L_{z}^{2}\left(\mathbb{R}^{n}\right)} .
$$

We now denote $\chi_{r, t}(x, \xi)=\left(x_{r, t}, \xi_{r, t}\right)$ as the time $r$ solution to the initial value problem

$$
\begin{gathered}
\frac{d x}{d s}=d_{\xi} p(x, s, \xi), \quad \frac{d \xi}{d s}=-d_{x} p(x, s, \xi), \\
(x(t), \xi(t))=(x, \xi) .
\end{gathered}
$$

This allows us to solve the transport equation and write

$$
\tilde{v}(x, t, \xi)=\left(T_{\lambda} v_{0}\right)\left(\chi_{0, t}(x, \xi)\right)+\int_{0}^{t} G_{r}\left(\chi_{r, t}(x, \xi)\right)+\tilde{F}_{r}\left(\chi_{r, t}(x, \xi)\right) d r,
$$

where $G_{r}(x, \xi)=G(x, r, \xi)$ and $\tilde{F}_{r}(x, \xi)=F(x, r, \xi)$. Since $T_{\lambda}^{*}$ is a left inverse for $T_{\lambda}$, this in turn implies that

$$
v(\cdot, t)=T_{\lambda}^{*}\left(T_{\lambda} v_{0} \circ \chi_{0, t}\right)+\int_{0}^{t} T_{\lambda}^{*}\left(G_{r} \circ \chi_{r, t}\right) d r+\int_{0}^{t} T_{\lambda}^{*}\left(\tilde{F}_{r} \circ \chi_{r, t}\right) d r .
$$

As in [10], we now define the operator $W_{r}$ mapping functions on $\mathbb{R}_{x, \xi}^{2 n}$ to $\mathbb{R}_{y, s}^{n+1}$ by

$$
\left(W_{r} f\right)(y, s)=T_{\lambda}^{*}\left(f \circ \chi_{r, s}\right)(y)
$$


Let $\widetilde{\Psi}_{\lambda}(\xi)$ denote a smooth cutoff identically 1 on the set in (4.5) and supported in a cone of slightly larger aperture in a set with $|\xi| \approx \lambda$. Since $T_{\lambda}$ is an isometry, in order to control the $L_{y^{\prime}, s}^{q} L_{y_{1}}^{2}$ norm of $v$, it will suffice to show that

$$
\widetilde{\Psi}_{\lambda}(D) W_{r}: L_{x, \xi}^{2}\left(\mathbb{R}^{2 n}\right) \rightarrow L_{y^{\prime}, s}^{q}\left(\mathbb{R}^{n-1} \times[-2,2] ; L_{y_{1}}^{2}(\mathbb{R})\right)
$$

with operator norm bounded by $\lambda^{\delta(q)}$ uniformly for $r \in[-2,2]$. By duality, this is equivalent to showing that

(4.11)

$\left\|\widetilde{\Psi}_{\lambda}(D) W_{r} W_{r}^{*} \widetilde{\Psi}_{\lambda}(D) F\right\|_{L_{z^{\prime}, t}^{q^{\prime}}\left(\mathbb{R}^{n-1} \times[-2,2] ; L_{z_{1}}^{2}(\mathbb{R})\right)} \lesssim \lambda^{2 \delta(q)}\|F\|_{L_{y^{\prime}, s}^{q}\left(\mathbb{R}^{n-1} \times[-2,2] ; L_{y_{1}}^{2}(\mathbb{R})\right)}$.

Next observe that we can characterize the action of the operator $W_{r} W_{r}^{*} \widetilde{\Psi}_{\lambda}(D)$ on a function $\widetilde{F}(z, t)$ as an integration of the function $\left(\widetilde{\Psi}_{\lambda} \widetilde{F}\right)(z, t)$ against a kernel $K(y, s ; z, t)$, where

$K(y, s ; z, t)=\lambda^{\frac{n}{2}} \int e^{i\langle\xi, y-x\rangle-i\left\langle\xi_{t, s}, z-x_{t, s}\right\rangle} g\left(\lambda^{\frac{1}{2}}(y-x)\right) g\left(\lambda^{\frac{1}{2}}\left(z-x_{t, s}\right)\right) \gamma\left(\lambda^{-1} \xi\right) d x d \xi$

and $\gamma$ is a smooth cutoff function. This is accomplished by a straightforward computation which uses the fact that $\chi_{r, s}$ is a measure-preserving diffeomorphism and that $\chi_{t, r} \circ \chi_{r, s}=\chi_{t, s}$. Observe also that by (4.7) we can choose $\gamma$ to be supported in a region slightly larger than that in (4.5). Hence by choosing $M$ sufficiently large in (2.14) we can assume that the support of $\gamma$ is contained in a cone with aperture that can be made arbitrarily small.

We now seek to show the following pair of estimates:

$$
\begin{gathered}
\left\|\int K(y, s ; z, t) f(z) d z\right\|_{L_{y}^{2}\left(\mathbb{R}^{n}\right)} \lesssim\|f\|_{L_{z}^{2}\left(\mathbb{R}^{n}\right)}, \\
\left\|\int K(y, s ; z, t) f(z) d z\right\|_{L_{y^{\prime} L_{y_{1}}^{\infty}\left(\mathbb{R}^{n}\right)}} \lesssim \lambda^{n-1}(1+\lambda|t-s|)^{-\frac{n-1}{2}}\|f\|_{L_{z^{\prime}}^{1} L_{z_{1}}^{2}\left(\mathbb{R}^{n}\right)} .
\end{gathered}
$$

Interpolation will then yield

$$
\left\|\int K(y, s ; z, t) f(z) d z\right\|_{L_{y^{\prime}}^{q} L_{y_{1}}^{2}} \lesssim \lambda^{(n-1)\left(1-\frac{2}{q}\right)}(1+\lambda|t-s|)^{-\frac{n-1}{2}\left(1-\frac{2}{q}\right)}\|f\|_{L_{z^{\prime}}^{q^{\prime}} L_{z_{1}}^{2}} .
$$

Since $\frac{n-1}{2}\left(1-\frac{2}{q}\right) \geq \frac{2}{q}=1-\frac{1}{q^{\prime}}+\frac{1}{q}$ whenever $q \geq \frac{2(n+1)}{n-1}$, we then have

$$
\left\|\int K(y, s ; z, t) f(z) d z\right\|_{L_{y^{\prime}}^{q} L_{y_{1}}^{2}\left(\mathbb{R}^{n}\right)} \lesssim \lambda^{2 \delta(q)}|t-s|^{-1+\frac{1}{q^{\prime}}-\frac{1}{q}}\|f\|_{L_{z^{\prime}}^{q^{\prime}} L_{z_{1}}^{2}\left(\mathbb{R}^{n}\right)} .
$$

The estimates (4.11) will then follow by the Hardy-Littlewood-Sobolev inequality of fractional integration.

To establish (4.13), we observe that

$$
\int K(y, s ; z, t)\left(\Gamma_{\lambda} f\right)(z) d z=T_{\lambda}^{*}\left(\left(T_{\lambda} \Gamma_{\lambda} f\right) \circ \chi_{t, s}\right)(y) .
$$

The inequality then follows since $\chi_{t, s}$ is a symplectic, measure-preserving diffeomorphism and by the $L^{2}$ mapping properties of $T_{\lambda}$.

Most of the work is thus involved in establishing (4.14). The main idea here is to obtain pointwise estimates on the $K(y, s ; z, t)$ that will imply the desired mapping properties. These inequalities will reflect the decay of $K$ away from the light cone 
as well as the dispersive properties of $K$. Additionally, since $\gamma$ is localized to a cone about the negative $\xi_{1}$ axis, $K(y, s ; z, t)$ should decay rapidly when $\left|y^{\prime}-z^{\prime}\right| \gg$ $\left|y_{1}-z_{1}\right|$. Therefore we should only need to examine properties of the part of the light cone whose projection down to the spatial variables lies in the complement of this set.

To accomplish this, we begin by establishing the existence of functions $\Phi_{z, t}^{ \pm}(y)$, defined when $\mp\left(y_{1}-z_{1}\right) \gg\left|y^{\prime}-z^{\prime}\right|$, such that $\left(y, \Phi_{z, t}^{+}(y)\right)$ (or $\left.\left(y, \Phi_{z, t}^{-}(y)\right)\right)$ is the graph of the forward (backward) light cone centered at $(z, t)$. We then seek to establish the pointwise estimate

$$
|K(y, s ; z, t)| \leq C_{N} \lambda^{n}(1+\lambda|s-t|)^{-\frac{n-1}{2}}(1+\lambda V(y, s ; z, t))^{-N},
$$

where $V$ is a non-negative measurable function. $V$ will have the property that

$$
V(y, s ; z, t)=\left|s-\Phi_{z, t}^{ \pm}(y)\right|
$$

for $|s|,|t| \leq 2,|y-z| \leq \frac{5}{2}$, and $y-z \in \widetilde{\Omega}_{ \pm}$, with $\widetilde{\Omega}_{+}$(resp. $\left.\widetilde{\Omega}_{-}\right)$a small cone about the negative (resp. positive) $x_{1}$-axis to be determined later. Here $\Phi_{z, t}^{+}$is used in the estimate if $s>t$, and $\Phi_{z, t}^{-}$is used otherwise. For $(y, s ; z, t)$ not in this domain, we will have $V(y, s ; z, t)=|y-z|$. As we will see, $\partial_{y_{1}} \Phi_{z, t}^{ \pm}(y)$ is uniformly bounded from below on the region where it is defined, allowing us to get suitable control over

$$
\sup _{z} \int|K(y, s ; z, t)| d y_{1}
$$

by changing variables. Hence the estimate (4.14) will follow by Schur's Lemma and the symmetry of $(y, s)$ and $(z, t)$ in the definition of $K$.

The estimates (4.15) will follow by a scaling argument of Smith [10. Assume without loss of generality that $s=0$ and $t \in[0,2]$. Let $\varepsilon$ be a scaling factor such that $\lambda^{-1} \leq \varepsilon \leq 1$ and consider the scaled kernel

$$
K_{\varepsilon}(y ; z, t)=\varepsilon^{n} K(\varepsilon y, 0 ; \varepsilon z, \varepsilon t) .
$$

Observe that if $\left(\tilde{x}_{t}, \tilde{\xi}_{t}\right)$ is an integral curve of the Hamiltonian flow determined by $p(x, t, \xi)$, then $\left(x_{t}, \xi_{t}\right)=\left(\varepsilon^{-1} \tilde{x}_{\varepsilon t}, \varepsilon \tilde{\xi}_{\varepsilon t}\right)$ is an integral curve of the flow determined by the Hamiltonian function

$$
H(x, t, \xi)=p(\varepsilon x, \varepsilon t, \xi) .
$$

This allows us to set $R=\varepsilon \lambda^{\frac{1}{2}}, \mu=\varepsilon \lambda$ and make a change of variables $(x, \xi) \mapsto$ $\left(\varepsilon x, \varepsilon^{-1} \xi\right)$ putting $K_{\varepsilon}$ in the form

$$
K_{\varepsilon}(y ; z, t)=R^{n} \int e^{i\langle\xi, y-x\rangle-i\left\langle\xi_{t}, z-x_{t}\right\rangle} g(R(y-x)) g\left(R\left(z-x_{t}\right)\right) \gamma\left(\mu^{-1} \xi\right) d x d \xi
$$

with $\left(x_{0}, \xi_{0}\right)=(x, \xi)$. We will not need to refer to the first component of $x_{t}$ specifically, so for the remainder of this paper $x_{1}(x, \xi)$ will refer to a vector, namely the projection of the time 1 solution to Hamilton's equations down to the $x$ variables with initial conditions $(x, \xi)$. The inequalities (4.15) are now a consequence of the following lemma, which is akin to Lemma 3.4 in [10].

Lemma 4.1. Let $\Lambda_{z}$ denote the $s=0$ time slice of the backward light cone centered at $(1, z)$ and let $d\left(y, \Lambda_{z}\right)$ denote the distance to $\Lambda_{z}$. Then for some $C_{0}$ sufficiently 
large and $\mu \geq C_{0}$, we have the following pointwise estimates on $K_{\varepsilon}(y ; z, 1)$ :

$$
\left|K_{\varepsilon}(y ; z, 1)\right| \leq \begin{cases}C_{N} \mu^{\frac{n+1}{2}}\left(1+\mu d\left(y, \Lambda_{z}\right)\right)^{-N}, & y-z \in \widetilde{\Omega}_{-}, \\ C_{N, N^{\prime}} \mu^{-N^{\prime}}(1+\mu|y-z|)^{-N}, & y-z \notin \widetilde{\Omega}_{-},\end{cases}
$$

with constants $C_{N}, C_{N, N^{\prime}}$ independent of $\mu \geq C_{0}$. Additionally, when $\mu=1$ we then have that for all $t \in\left[0, C_{0}\right]$

$$
\left|K_{\varepsilon}(y ; z, t)\right| \leq C_{N}(1+|y-z|)^{-N} .
$$

To see that this lemma implies (4.15), observe that when $t \geq C_{0} \lambda^{-1}$, we can set $\varepsilon=t$ and rescale (4.17) to get that

$$
|K(y, 0 ; z, t)| \leq \begin{cases}C_{N} \lambda^{n}(1+\lambda t)^{-\frac{n-1}{2}}\left(1+\lambda d\left(y, \Lambda_{z, t}\right)\right)^{-N}, & y-z \in \widetilde{\Omega}_{-}, \\ C_{N}(1+\lambda t)^{-\frac{n-1}{2}}(1+\lambda|y-z|)^{-N}, & y-z \notin \widetilde{\Omega}_{-},\end{cases}
$$

where $\Lambda_{z, t}$ denotes the time 0 slice of the light cone centered at $(z, t)$. It is clear this establishes the desired estimate in (4.15) when $t \geq C_{0} \lambda^{-1}$ and $y-z \notin \widetilde{\Omega}_{-}$. When $y-z \in \widetilde{\Omega}_{-}$and $|y-z| \geq \frac{5}{2}$ then $y$ is uniformly at distance $\frac{1}{8}$ away from the light cone for $t \in[0,2]$, and hence we can conclude that $d\left(y, \Lambda_{z, t}\right) \gtrsim 1+|y-z|$. In

the case where $y-z \in \widetilde{\Omega}_{-}$and $|y-z| \leq \frac{5}{2}, y$ is in the domain of $\Phi^{-}$, and hence we will be able to use the approximation (5.16) below to obtain $\left|\Phi_{z, t}^{-}(y)\right| \approx d\left(y, \Lambda_{z, t}\right)$ so that (4.15) holds.

In the case where $t \leq C_{0} \lambda^{-1}$, we set $\varepsilon=\lambda^{-1}$ so that rescaling (4.18) yields the desired inequalities in (4.15).

\section{Regularity of the Hamiltonian flow}

In this section we present the arguments needed to prove Lemma 4.1 The main idea here is to characterize integration against $K_{\varepsilon}$ as being equivalent to the action of the adjoint of some Fourier integral operator. This means that we wish to find a phase function $\varphi_{t}(z, \eta)$ and symbol $a_{t}(z, \eta)$ (supported in a cone about the negative $\eta_{1}$ axis) such that

$$
K_{\varepsilon}(y ; z, t)=\int e^{i\langle y, \eta\rangle-i \varphi_{t}(z, \eta)} a_{t}(z, \eta) d \eta .
$$

Once this is accomplished, we will be able to use a second dyadic decomposition of $\eta$-space to get the desired estimates on $K_{\varepsilon}$.

We begin with a lemma due to H. Smith 10 concerning the integral curves $\left(x_{t}, \xi_{t}\right)$ of the Hamiltonian flow induced by $H(x, t, \xi)$, that is, the solutions to the system

$$
\begin{aligned}
& \frac{d x}{d t}=H_{\xi}(x, t, \xi), \\
& \frac{d \xi}{d t}=-H_{x}(x, t, \xi) .
\end{aligned}
$$

Lemma 5.1. Let $\left(x_{t}(x, \xi), \xi_{t}(x, \xi)\right)$ denote the solution to (5.1) with initial conditions $x(0)=x, \xi(0)=\xi$. Then for $-C_{0} \leq t \leq C_{0}$ and $\xi \in\left\{\xi \in \mathbb{S}^{n-1}:-\xi_{1} \geq 7\left|\xi^{\prime}\right|\right\}$ we have

$$
\left|x_{t}-\left(x+t \frac{\left(\xi_{1},-\xi^{\prime}\right)}{\sqrt{\xi_{1}^{2}-\left|\xi^{\prime}\right|^{2}}}\right)\right|+\left|\xi_{t}-\xi\right| \ll t
$$


and

$$
\left|d_{x} x_{t}-I\right|+\left|d_{x} \xi_{t}\right|+\left|d_{\xi} x_{t}-\int_{0}^{t} d_{\xi}^{2} H\left(s, x_{s}, \xi_{s}\right) d s\right|+\left|d_{\xi} \xi_{t}-I\right| \ll 1 .
$$

We have estimates on higher order derivatives with $|\alpha|+|\beta| \geq 2$ and $0<t<C_{0}$, $|\xi|=1$. For $R \leq 1$ :

$$
\begin{array}{r}
\left|\partial_{x}^{\beta} \partial_{\xi}^{\alpha} x_{t}\right| \lesssim \begin{cases}1, & |\beta|=0, \\
\varepsilon, & |\beta|=1, \\
\varepsilon R^{|\beta|-2}, & |\beta| \geq 2,\end{cases} \\
\left|\partial_{x}^{\beta} \partial_{\xi}^{\alpha} \xi_{t}\right| \lesssim \begin{cases}\varepsilon, & |\beta|=0, \\
\varepsilon R^{|\beta|-1}, & |\beta| \geq 1 .\end{cases}
\end{array}
$$

For $R \geq 1$ :

$$
\begin{aligned}
& \left|\partial_{x}^{\beta} \partial_{\xi}^{\alpha} x_{t}\right| \lesssim \begin{cases}1+\varepsilon R^{|\alpha|-1}, & |\beta|=0, \\
\varepsilon R^{|\alpha|+|\beta|-1}, & |\beta| \geq 1,\end{cases} \\
& \left|\partial_{x}^{\beta} \partial_{\xi}^{\alpha} \xi_{t}\right| \lesssim \varepsilon R^{|\alpha|+|\beta|-1} .
\end{aligned}
$$

The proof of this lemma relies only on the estimates

$$
\int_{-C_{0}|\xi|=1}^{C_{0}} \sup _{x} \sup _{x}\left|\partial_{t, x}^{\beta} \partial_{\xi}^{\alpha} H(t, x, \xi)\right| d t \leq \begin{cases}C_{\alpha}, & |\beta|=0, \\ C_{\alpha} \varepsilon, & |\beta|=1, \\ C_{\alpha, \beta} \varepsilon R^{|\beta|-2}, & |\beta| \geq 2,\end{cases}
$$

which follow from the definition of $H(t, x, \xi)$ and (4.3), (4.4). Hence the proof in Lemma 4.1 of [10] works here as well. We now provide a sketch of these arguments.

Proof. First observe that (5.2) follows from a straightforward estimate involving (4.1). To handle first order derivatives in (5.3) we define the matrix

$$
M=\left[\begin{array}{cc}
d_{x} d_{\xi} H & d_{\xi} d_{\xi} H \\
-d_{x} d_{x} H & -d_{\xi} d_{x} H
\end{array}\right]
$$

and differentiate Hamilton's equations to obtain

$$
\partial_{t}\left[\begin{array}{l}
d_{x} x_{t} \\
d_{x} \xi_{t}
\end{array}\right]=M\left(t, x_{t}, \xi_{t}\right) \cdot\left[\begin{array}{l}
d_{x} x_{t} \\
d_{x} \xi_{t}
\end{array}\right], \quad \partial_{t}\left[\begin{array}{l}
d_{\xi} x_{t} \\
d_{\xi} \xi_{t}
\end{array}\right]=M\left(t, x_{t}, \xi_{t}\right) \cdot\left[\begin{array}{l}
d_{\xi} x_{t} \\
d_{\xi} \xi_{t}
\end{array}\right] .
$$

Only the upper right hand block in $M$ has norm $\approx 1$ in $L_{t}^{1}\left(-C_{0}, C_{0}\right)$; the rest have norm $\ll 1$ when integrated in time. Also, when $t=0$, we have $d_{x} x_{t}=d_{\xi} \xi_{t}=I$ and $d_{\xi} x_{t}=d_{x} \xi_{t}=0$, so that an application of Gronwall's inequality yields (5.3).

For higher order derivatives we write

$$
\partial_{t}\left[\begin{array}{c}
\partial_{x}^{\beta} \partial_{\xi}^{\alpha} x_{t} \\
\partial_{x}^{\beta} \partial_{\xi}^{\alpha} \xi_{t}
\end{array}\right]=M\left(t, x_{t}, \xi_{t}\right) \cdot\left[\begin{array}{c}
\partial_{x}^{\beta} \partial_{\xi}^{\alpha} x_{t} \\
\partial_{x}^{\beta} \partial_{\xi}^{\alpha} \xi_{t}
\end{array}\right]+\left[\begin{array}{c}
E_{1} \\
E_{2}
\end{array}\right]
$$

where $E_{1}, E_{2}$ are a sum of terms, each of which is the product of derivatives of $H$ with derivatives of $x_{t}, \xi_{t}$ that have lower order than $\alpha+\beta$. The remaining estimates on higher order derivatives then follow by considering each case $R \geq 1$, $R \leq 1$ separately, and proceeding by an induction argument which uses (5.8). See Lemma 4.1 of [10] for complete details. 
By (5.3) we have that for any $\xi \in \mathbb{S}^{n-1}$ such that $-\xi_{1} \geq 7\left|\xi^{\prime}\right|$ and $t \in\left[-C_{0}, C_{0}\right]$, the map $x \mapsto x_{t}(x, \xi)$ is a local diffeomorphism on $\mathbb{R}^{n}$ with uniform bounds on its differential. It is also a proper map since $\left|x_{t}(x, \xi)-x\right| \leq 5$. This implies that it is an open and closed map as well as being a covering map. By connectivity, its image is all of $\mathbb{R}^{n}$. Additionally, since $\mathbb{R}^{n}$ is simply connected, it is also an injective map. Thus the map is a diffeomorphism and we let $z \mapsto \bar{x}_{t}(z, \xi)$ denote the inverse map.

We now define the generating function $\varphi_{t}(z, \xi)$ by

$$
\varphi_{t}(z, \xi)=\left\langle\xi, \bar{x}_{t}(z, \xi)\right\rangle
$$

which possesses the following properties for $\xi$ such that $|\xi|=1$ and $-\xi_{1} \geq 7\left|\xi^{\prime}\right|$ :

$$
d_{z} \varphi_{t}(z, \xi)=\xi_{t}\left(\bar{x}_{t}(z, \xi), \xi\right), \quad d_{\xi} \varphi_{t}(z, \xi)=\bar{x}_{t}(z, \xi)
$$

To see this, set $y=\bar{x}_{t}(z, \xi), \eta=\xi_{t}\left(\bar{x}_{t}(z, \xi), \xi\right)$ so that $\varphi_{t}(z, \xi)=\sum_{i} y_{i} \xi_{i}$. Since $(x, \xi) \mapsto\left(x_{t}, \xi_{t}\right)$ is a canonical transformation we have

$$
d y \wedge d \xi=d z \wedge d \eta
$$

We then substitute the following into this equality:

$$
d y_{i}=\sum_{j=1}^{n} \partial_{z_{j}} y_{i} d z_{j}+\partial_{\xi_{j}} y_{i} d \xi_{j} \quad \text { and } \quad d \eta_{i}=\sum_{j=1}^{n} \partial_{z_{j}} \eta_{i} d z_{j}+\partial_{\xi_{j}} \eta_{i} d \xi_{j} .
$$

This now yields $\partial_{z_{j}} y_{i}=\partial_{\xi_{i}} \eta_{j}$ for any $i, j$ and $\partial_{\xi_{j}} y_{i}=0$ when $i \neq j$. Since $y$ is homogeneous of degree 0 in $\xi$ and $\eta$ is homogeneous of degree 1 in $\xi$, we now have the desired equalities

$$
\begin{gathered}
\partial_{z_{j}}\left(\sum_{i} \xi_{i} y_{i}\right)=\sum_{i} \xi_{i} \partial_{z_{j}} y_{i}=\sum_{i} \xi_{i} \partial_{\xi_{i}} \eta_{j}=\eta_{j}, \\
\partial_{\xi_{j}}\left(\sum_{i} \xi_{i} y_{i}\right)=y_{j}+\sum_{i} \xi_{i} \partial_{\xi_{j}} y_{i}=y_{j}+\sum_{i} \xi_{i} \partial_{\xi_{i}} y_{j}=y_{j} .
\end{gathered}
$$

Also, by Lemma 4.3 and Lemma 4.4 of [10], we have that for $|\xi|=1$ and $|t| \leq C_{0}$,

$$
\left|\partial_{z}^{\beta} \partial_{\xi}^{\alpha} d_{z}^{2} \varphi_{t}(z, \xi)\right| \leq C_{\alpha, \beta} R^{|\beta|}(1+R)^{|\alpha|}
$$

with $C_{\alpha, \beta}$ independent of the choice of $\xi, t$. This follows by first observing that given the inverse function theorem, $\bar{x}_{t}(z, \xi)$ enjoys the same higher order derivative estimates (5.4), (5.6) that $x_{t}(x, \xi)$ satisfies from Lemma 5.1. The inequality (5.10) then follows by combining the first equality in (5.9) with such estimates.

In order to characterize $K_{\varepsilon}$ as the integral kernel of the adjoint of a Fourier integral operator, we take the Fourier transform of the first factor of $g$ in (4.16) and write

$$
\begin{aligned}
K_{\varepsilon}(y ; z, t) & =\int e^{i\langle\eta, y-x\rangle-i\left\langle\xi_{t}, z-x_{t}\right\rangle} h\left(R^{-1}(\eta-\xi)\right) g\left(R\left(z-x_{t}\right)\right) \gamma\left(\mu^{-1} \xi\right) d x d \xi d \eta \\
& =\int e^{i\langle y, \eta\rangle-i \varphi_{t}(z, \eta)} a_{t}(z, \eta) d \eta
\end{aligned}
$$

where $h=\widehat{g}$ and

$$
\begin{aligned}
& a_{t}(z, \eta)=\int e^{i \varphi_{t}(z, \eta)-i\langle x, \eta\rangle-i\left\langle\xi_{t}(x, \xi), z-x_{t}(x, \xi)\right\rangle} \\
& \cdot h\left(R^{-1}(\eta-\xi)\right) g\left(R\left(z-x_{t}(x, \xi)\right)\right) \gamma\left(\mu^{-1} \xi\right) d x d \xi
\end{aligned}
$$


We note that since $\gamma$ is compactly supported in a cone about the negative $\xi_{1}$-axis, we have that for $\mu$ sufficiently large, $a_{t}(z, \cdot)$ must be supported in a set where $|\eta| \approx \mu$ inside a cone of slightly larger aperture about the negative $\eta_{1}$-axis. Observe also that by choosing $M$ large in (2.14) and $\mu$ sufficiently large, we can assume that the support of $a_{t}(z, \cdot)$ lies in a cone of arbitrarily small (but fixed) aperture.

Citing Theorem 4.5 in [10] we have that for $t \in\left[-C_{0}, C_{0}\right]$ and $\mu \geq 1, a_{t}(z, \eta)$ satisfies the estimates

$$
\left|\partial_{\eta}^{\alpha}\left\langle\eta, d_{\eta}\right\rangle^{k} a_{t}(z, \eta)\right| \leq C_{\alpha, k}\left(1+R^{2}\right)^{\frac{|\alpha|}{2}} \mu^{-|\alpha|} .
$$

We pause to remark that the proof of (5.13) depends on estimates on the derivatives of $x_{t}(x, \xi), \xi_{t}(x, \xi), \bar{x}_{t}(z, \xi)$, and $\varphi_{t}(z, \xi)$ but not on the specific geometry of the flow induced by $H(x, t, \xi)$. We sketch the argument and provide key details of Smith's proof, but refer the reader to [10] for complete details.

Proof sketch of (5.13). First consider the case $k=0$. We first define a function $p(x, z, \xi, \eta)$ as a symbol of size $A$ if it satisfies

$$
\left|\partial_{\eta}^{\theta} \partial_{\xi}^{\alpha} p(x, z, \xi, \eta)\right| \lesssim A \cdot\langle R\rangle^{|\alpha|+|\theta|} \mu^{-|\alpha|-|\theta|} .
$$

The proof then follows by an inductive argument, considering more general integrals

$$
\begin{aligned}
\tilde{a}_{t}(z, \eta)=\int e^{i \varphi_{t}(z, \eta)-i\langle x, \eta\rangle-i\left\langle\xi \xi_{t}(x, \xi), z-x_{t}(x, \xi)\right\rangle} & \\
& \cdot p(x, z, \xi, \eta) \tilde{h}\left(R^{-1}(\eta-\xi)\right) \tilde{g}\left(R\left(z-x_{t}(x, \xi)\right)\right) \tilde{\gamma}\left(\mu^{-1} \xi\right) d x d \xi .
\end{aligned}
$$

Here $\operatorname{supp}(\tilde{h}) \subset B_{1}(0), \tilde{\gamma}$ is compactly supported near the negative $\xi_{1}$-axis, and $\tilde{g}$ is of Schwartz class so that they are slight modifications of $h, \gamma$, and $g$, respectively. The main idea is to see that $\partial_{\eta_{j}} \tilde{a}_{t}(z, \eta)$ yields an integral with essentially the same form as $\tilde{a}_{t}(z, \eta)$ except with a symbol $p$ of size $\langle R\rangle \mu^{-1}$.

Observe that the action of the operator $\partial_{\eta_{j}}$ on the $h$ factor in the integrand is equivalent to the action of $-\partial_{\xi_{j}}$ on that same factor. Therefore, integration by parts gives that the effect of $-i \partial_{\eta_{j}}$ on $\tilde{a}_{t}(z, \eta)$ is the same as multiplying the integrand by the symbol

$$
\partial_{\eta_{j}} \varphi_{t}(z, \eta)-x_{j}-\partial_{\xi_{j}}\left\langle\xi_{t}(x, \xi), z-x_{t}(x, \xi)\right\rangle-R \partial_{\xi_{j}} x_{t}(x, \xi)
$$

modulo terms that exhibit better behavior. We first observe that by homogeneity and (5.6), (5.4), $R \partial_{\xi_{j}} x_{t}(x, \xi)$ yields a symbol of size $R \mu^{-1}$. Second, we observe that we can replace $\partial_{\eta_{j}} \varphi_{t}(z, \eta)$ by $\partial_{\xi_{j}} \varphi_{t}(z, \xi)$, as the second equality in (5.9) gives

$$
d_{\eta} \varphi_{t}(z, \eta)-d_{\xi} \varphi_{t}(z, \xi)=\int_{0}^{1} d_{\xi} \bar{x}_{t}(z, s \eta+(1-s) \xi) \cdot(\eta-\xi) d s .
$$

After changing the form of $\tilde{h}$ this yields a symbol of size $R \mu^{-1}$.

We now use the fact that $\bar{x}_{t}$ is the inverse of $x_{t}$ and the first equality in (5.9) to obtain $\xi_{t}(x, \xi)=d_{z} \varphi_{t}\left(x_{t}(x, \xi), \xi\right)$ and $\langle x, \xi\rangle=\varphi_{t}\left(x_{t}(x, \xi), \xi\right)$. Hence we are left to estimate

$$
\partial_{\xi_{j}}\left[\varphi_{t}(z, \xi)-\varphi_{t}\left(x_{t}(x, \xi), \xi\right)-\left(z-x_{t}(x, \xi)\right) \cdot\left(d_{z} \varphi_{t}\right)\left(x_{t}(x, \xi), \xi\right)\right] .
$$

Replacing the expression in brackets by its second order Taylor remainder term in $z$, it can be seen that by (5.10) that this yields a symbol of size $\langle R\rangle \mu^{-1}$ after changing the form of $\tilde{g}$. 
To handle the radial derivative estimates, we use the fact that

$$
\left\langle\eta, d_{\eta}\right\rangle^{k} a(z, \eta)=\left.\left(r \partial_{r}\right)^{k} a(z, r \eta)\right|_{r=1} .
$$

Thus by changing variables $\xi \rightarrow r \xi$ in $a_{t}(z, r \eta)$ and using homogeneity we obtain

$$
\begin{aligned}
a_{t}(z, r \eta)=r^{n} \int e^{i r\left[\varphi_{t}(z, \eta)-i\langle x, \eta\rangle-i\left\langle\xi_{t}(x, \xi), z-x_{t}(x, \xi)\right\rangle\right]} & \\
& h\left(r R^{-1}(\eta-\xi)\right) g\left(R\left(z-x_{t}(x, \xi)\right)\right) \beta\left(r \mu^{-1} \xi\right) d x d \xi .
\end{aligned}
$$

When applied to $h$ or $\beta$, the operator $\partial_{r}$ just changes the form of the function. Hence we just need to see that the phase

$$
\varphi_{t}(z, \eta)-\langle x, \eta\rangle-\left\langle\xi_{t}(x, \xi), z-x_{t}(x, \xi)\right\rangle
$$

is essentially a symbol of size 1 . As before,

$$
\varphi_{t}(z, \xi)-\langle x, \xi\rangle-\left\langle\xi_{t}(x, \xi), z-x_{t}(x, \xi)\right\rangle
$$

can be seen to be a symbol of size 1 by replacing it with its second order Taylor remainder. Hence it suffices to consider

$$
\begin{aligned}
\varphi_{t}(z, \eta)-\varphi_{t}(z, \xi)-\langle x, \eta\rangle+\langle x, \xi\rangle & =\int_{0}^{1}\left\langle d_{\xi} \varphi_{t}(z, s \eta+(1-s) \xi)-x, \eta-\xi\right\rangle d s \\
& =\int_{0}^{1}\left\langle\bar{x}_{t}(z, s \eta+(1-s) \xi)-x, \eta-\xi\right\rangle .
\end{aligned}
$$

By writing

$\bar{x}_{t}(z, s \eta+(1-s) \xi)-x=\left(\bar{x}_{t}(z, s \eta+(1-s) \xi)-\bar{x}_{t}(z, \xi)\right)+\left(\bar{x}_{t}(z, \xi)-\bar{x}_{t}\left(x_{t}(x, \xi), \xi\right)\right)$

we see that we can replace each difference in parentheses by its first order Taylor remainder. Using derivative estimates and altering the form of $\tilde{g}$ and $\tilde{h}$ we see that this yields a symbol of size 1 .

We now introduce some technical notation. For $i=0,1,2,3,4,5$ we let $\Omega_{i}^{+}$ denote small open cones about the negative $\xi_{1}$-axis such that for any $z \in \mathbb{R}^{n}$,

$$
\operatorname{supp}\left(a_{t}(z, \cdot)\right) \subset \Omega_{0}^{+} \subset \Omega_{1}^{+} \subset \Omega_{2}^{+} \subset \Omega_{3}^{+} \subset \Omega_{4}^{+} \subset \Omega_{5}^{+},
$$

and the aperture of $\Omega_{i}^{+}$is strictly greater than that of $\Omega_{i-1}^{+}$. As with other conic sets of this type we assume that their aperture can be made arbitrarily small by choosing $M$ in (2.14) and $\mu$ large. We also let

$$
\Omega_{i}^{-}=\left\{\xi:\left(-\xi_{1}, \xi^{\prime}\right) \in \Omega_{i}^{+}\right\} .
$$

Next we construct and analyze the $\Phi_{z, t}^{ \pm}$function introduced in the previous section. Let

$$
\Lambda_{i}^{ \pm}=\bigcup_{ \pm t \in[0,2)} \bigcup_{\xi \in \Omega_{i}^{+}}\left(x_{t}(0, \xi), t\right) \quad \text { and } \quad \Lambda_{i}^{ \pm, t}=\bigcup_{\xi \in \Omega_{i}^{+}}\left(x_{t}(0, \xi), t\right)
$$

be sections of the light cone or time slices of the light cone defined by $H$ centered at the origin. We define $\Lambda^{ \pm}\left(\Lambda^{ \pm, t}\right)$ similarly as the full (time slice of the) light cone at 0 by removing the condition $\xi \in \Omega_{i}^{+}$above and replacing it with $\xi \in \mathbb{R}^{n} \backslash\{0\}$. We now have the following lemma, which is akin to Lemma 4.2 in [10]. 
Lemma 5.2. $\Lambda_{5}^{ \pm}$is the graph $s=\Phi^{ \pm}(y)$ of a function $\Phi^{ \pm}(y)$ defined on a conic neighborhood containing $\Omega_{4}^{ \pm} \cap\{z:|z| \leq 2\}$. The function $\Phi^{ \pm}(y)$ has the following properties:

$$
\left|\Phi^{ \pm}(y) \mp \sqrt{y_{1}^{2}-\left|y^{\prime}\right|^{2}}\right| \ll|y|, \quad\left|d_{y} \Phi^{ \pm}(y) \mp \frac{\left(y_{1},-y^{\prime}\right)}{\sqrt{y_{1}^{2}-\left|y^{\prime}\right|^{2}}}\right| \ll 1, \quad\left|d_{y}^{2} \Phi^{ \pm}(y)\right| \lesssim \frac{1}{|y|} .
$$

Additionally, for $\pm s \in(0,2), \Lambda_{i}^{ \pm, s}$ is a convex hypersurface in $\mathbb{R}^{n}$, and for $x, y \in$ $\Lambda_{5}^{ \pm, s}$ we have

$$
\left|\frac{d_{x} \Phi^{ \pm}(x)}{\left|d_{x} \Phi^{ \pm}(x)\right|}-\frac{d_{y} \Phi^{ \pm}(y)}{\left|d_{y} \Phi^{ \pm}(y)\right|}\right| \approx \pm s^{-1}|x-y| .
$$

Lastly, if $y \in \Omega_{4}^{ \pm} \cap\left\{z:|z| \leq \frac{5}{2}\right\}$ and $\pm s \in[0,2)$, then

$$
d\left(y, \Lambda_{5}^{ \pm, s}\right) \approx\left|s-\Phi^{ \pm}(y)\right| .
$$

Proof. In what follows, we drop the ' \pm ' and prove the claim for the case corresponding to the forward light cone, as the case of the backward light cone is similar. As in the proof of Theorem 4.2 of [10], we will prove the estimates in (5.14) for $\widetilde{\Lambda}_{5}$, the part of $\Lambda_{5}$ corresponding to $\frac{1}{2}<t<\frac{5}{2}$. A scaling argument over smaller dyadic intervals will handle the remaining part. Given positive numbers $r_{1}, r_{2}>0$, let $A_{\left(r_{1}, r_{2}\right)}=\left\{z: r_{1}<|z|<r_{2}\right\}$. For $z$ in the polar section $\Omega_{5} \cap A_{\left(\frac{1}{2}, \frac{5}{2}\right)}$, let $z=r \omega$, where $(r, \omega) \in\left(\frac{1}{2}, \frac{5}{2}\right) \times \mathbb{S}^{n-1} \cap \Omega_{5}$, and set

$$
y(z)=x_{r}(0, \omega), \quad \xi(z)=\xi_{r}(0, \omega) .
$$

Let $\Psi(\xi)=\frac{\left(\xi_{1},-\xi^{\prime}\right)}{\sqrt{\xi_{1}^{2}-\left|\xi^{\prime}\right|^{2}}}, T=\operatorname{diag}(1,-1, \ldots,-1)$ be an $n \times n$ matrix, $\widetilde{\Pi}(\xi)=$ $D \Psi(\xi)=\frac{T}{\left(\xi_{1}^{2}-\left|\xi^{\prime}\right|^{2}\right)^{\frac{1}{2}}}-\frac{(T \xi) \cdot(T \xi)^{T}}{\left(\xi_{1}^{2}-\left|\xi^{\prime}\right|^{2}\right)^{\frac{3}{2}}}$, and $\Pi_{\omega}^{\perp}$ denote orthogonal projection onto the subspace normal to $\omega$. We then have that as a consequence of (5.3)

$$
\left|\partial_{r} y-\Psi(\omega)\right| \ll 1, \quad\left|\partial_{\omega} y-r \widetilde{\Pi}(\omega)\right| \ll 1, \quad\left|\partial_{r} \xi\right| \ll 1, \quad\left|\partial_{\omega} \xi-\Pi_{\omega}^{\perp}\right| \ll 1,
$$

which implies that over $\Omega_{5} \cap A_{\left(\frac{1}{2}, \frac{5}{2}\right)}$,

$$
\|y(z)-r \Psi(\omega)\|_{C^{1}} \ll 1, \quad \quad\|\xi(z)-\omega\|_{C^{1}} \ll 1 .
$$

Hence the map $z \rightarrow y(z)$ is a $C^{1}$ diffeomorphism from $\Omega_{5} \cap A_{\left(\frac{1}{2}, \frac{5}{2}\right)}$ onto its image $y\left(\Omega_{5} \cap A_{\left(\frac{1}{2}, \frac{5}{2}\right)}\right)$.

Hence $\Phi(y)=r$ is a well defined function on $y\left(\Omega_{5} \cap A_{\left(\frac{1}{2}, \frac{5}{2}\right)}\right)$ such that $\{(y, \Phi(y))$ : $\left.y \in y\left(\Omega_{5} \cap A_{\left(\frac{1}{2}, \frac{5}{2}\right)}\right)\right\}=\widetilde{\Lambda}_{5}$. By choosing $c_{0}$ sufficiently small in (2.4), $y(z)$ is a small perturbation of the map $r \Psi(\omega)$, meaning we can also assume that for $r \in\left(\frac{1}{2}, \frac{5}{2}\right)$

$$
\begin{aligned}
y\left(\Omega_{1} \cap\{z:|z|\right. & =r\}) \subset \Omega_{2} \cap \Lambda^{r} \\
& \subset y\left(\Omega_{3} \cap\{z:|z|=r\}\right) \subset \Omega_{4} \cap \Lambda^{r} \subset y\left(\Omega_{5} \cap\{z:|z|=r\}\right)
\end{aligned}
$$

and that there is a positive distance between the boundary of any two of these sets. 
The first estimate in (5.14) now follows by simple pointwise approximations. Next observe that since the Hamiltonian flow of $H$ yields a symplectic transformation, we have $\xi \cdot \partial_{\omega} y=0$. As in [10], the gradient of $\Phi$ is now given by

$$
\left(d_{y} \Phi\right)(y(z))=\frac{\xi(z)}{H(y(z), r, \xi(z))}
$$

as $\xi \cdot \partial_{r} y=H(y, r, \xi)$. The expression on the right is $C^{1}$ close to

$$
\xi(z) / \sqrt{\xi_{1}(z)^{2}-\left|\xi(z)^{\prime}\right|^{2}}
$$

which is in turn $C^{1}$ close to $(T y) / \sqrt{y_{1}^{2}-\left|y^{\prime}\right|^{2}}$. The remaining estimates in (5.14) now follow for $\frac{1}{2}<|z|<\frac{5}{2}$.

Suppose that $y \in \Lambda_{5}^{1}$, so that $y=y(\omega)$ for some $\omega \in \Omega_{5}$. By (5.19), we have that

$$
\frac{d_{y} \Phi(y)}{\left|d_{y} \Phi(y)\right|}=\frac{\xi(\omega)}{|\xi(\omega)|}
$$

Since $\left|\frac{\partial \xi(\omega)}{\partial \omega}-\Pi_{\omega}^{\perp}\right| \ll 1$, we have that the map $\omega \mapsto \frac{\xi(\omega)}{|\xi(\omega)|}$ is a bilipschitz diffeomorphism from $\Omega_{5} \cap \mathbb{S}^{n-1}$ onto its image. $\frac{\xi(\omega)}{|\xi(\omega)|}$ is the unit normal to $\Lambda_{5}^{1}$, so this means that the Gauss map associated to $\Lambda_{5}^{1}$ is injective, implying that it is a convex hypersurface. Since $\omega \mapsto y(\omega)$ is also bilipschitz from $\mathbb{S}^{n-1} \cap \Omega_{5} \rightarrow \Lambda_{5}^{1}$, the estimate (5.15) follows for $s=1$. The general case is now a result of a scaling argument.

To show (5.16), we observe again that by scaling we can restrict our attention to the case $s=1$. Additionally, the claim follows easily when $y$ is uniformly distant from the section of the hyperbola

$$
\left\{x: x_{1}^{2}=1+\left|x^{\prime}\right|^{2}, x_{1}<0\right\} \cap \Omega_{4} .
$$

In other words, we can reduce the inequality to the case where

$$
y \in \Omega_{4} \cap A_{\left(\frac{3}{4}, \frac{5}{4}\right)} \subset y\left(\Omega_{5} \cap A_{\left(\frac{1}{2}, \frac{5}{2}\right)}\right) \quad \text { and } \quad\left|\sqrt{y_{1}^{2}-\left|y^{\prime}\right|^{2}}-1\right|<\tilde{c}_{0}
$$

for some small $\tilde{c}_{0}>0$. Let $x$ be a point on $\Lambda_{5}^{1}$ such that $|x-y|=d\left(y, \Lambda_{5}^{1}\right)$. Observe that by choosing the aperture of the $\Omega_{i}$ to be sufficiently small, we have that the restriction above implies that $|x-y| \leq 1$.

We now claim that

$$
\left|d\left(y, \Lambda_{5}^{1}\right)-\right|\langle T x, y-x\rangle|| \leq \frac{1}{2}|y-x| .
$$

Suppose that $\omega, \tilde{\omega}$ are such that $x=x_{1}(0, \omega)$ and $\tilde{\omega}=\xi_{1}(0, \omega) /\left|\xi_{1}(0, \omega)\right|$. By choosing $c_{0}$ in (2.4) and $\tilde{c}_{0}$ above sufficiently small, we can assume that $\omega \in \Omega_{5}$ and that $|\langle\tilde{\omega}, y-x\rangle|=|y-x|=d\left(y, \Lambda_{5}^{1}\right)$. By approximating $T x \approx \frac{\omega}{\sqrt{\omega_{1}^{2}-\left|\omega^{\prime}\right|^{2}}}$ and $\omega \approx \tilde{\omega}$, this now implies

$$
\begin{aligned}
|\langle T x, y-x\rangle| & \leq C c_{0}|y-x|+\left|\left\langle\frac{\omega}{\sqrt{\omega_{1}^{2}-\left|\omega^{\prime}\right|^{2}}}, y-x\right\rangle\right| \\
& \leq|\langle\tilde{\omega}, y-x\rangle|+\left(1-\frac{1}{\sqrt{\omega_{1}^{2}-\left|\omega^{\prime}\right|^{2}}}\right)|y-x|+\tilde{C} c_{0}|y-x| .
\end{aligned}
$$

By choosing the $\Omega_{i}$ to have a sufficiently small angle about the negative $\xi_{1}$-axis, this means that

$$
|\langle T x, y-x\rangle|-d\left(y, \Lambda_{5}^{1}\right) \leq \frac{1}{2}|y-x| .
$$


The other requisite inequality needed in (5.20) follows by similar reasoning. Since

$$
|\Phi(y)-1|=\left|(y-x) \cdot \int_{0}^{1}\left(d_{y} \Phi\right)(x+(1-s)(y-x)) d s\right|,
$$

straightforward estimates on the integral involving the previous inequality and (5.14) now yield (5.16) for $s=1$.

We are now prepared to prove the desired estimates (4.17) and (4.18).

Proof of Lemma 4.1. We first observe that the estimate (4.18) follows by the proof of the analogous estimate in Lemma 3.4 of [10]. Indeed, we can write

$$
K_{\varepsilon}(y ; z, t)=\int e^{i\langle y-z, \eta\rangle}\left(e^{i\langle z, \eta\rangle-i \varphi_{t}(z, \eta)} a_{t}(z, \eta)\right) d \eta .
$$

Since $\mu=1$ implies $R \leq 1$, (5.4) and the observations above give

$$
\left|\partial_{\eta}^{\beta}\left(\bar{x}_{t}(z, \eta)-z\right)\right| \leq C_{\beta}
$$

for any $\beta \geq 0$. A simple integration by parts argument establishes the desired estimates.

We now prove (4.17), assuming $z=0$. A translation of coordinates will handle the case of arbitrary $z \in \mathbb{R}^{n}$. As in the analogous lemma from [10, we use a second dyadic decomposition to write $a_{1}(0, \eta)=\sum_{\nu} a^{\nu}(0, \eta)$, where $a^{\nu}(0, \eta)$ is supported in a cone of the form

$$
\left\{\eta:|\eta /| \eta\left|-\omega_{\nu}\right| \leq \mu^{-\frac{1}{2}}\right\}
$$

The collection $\left\{\omega_{\nu}\right\}_{\nu}$ can be taken so that it is contained in $\Omega_{1}$. We may also assume it is equally spaced distance $\mu^{-\frac{1}{2}}$ apart. By the estimates (5.13) on $a_{1}(0, \eta)$ this yields

$$
\left|\left\langle\omega_{\nu}, \partial_{\eta}\right\rangle^{j} \partial_{\eta}^{\beta} a^{\nu}(0, \eta)\right| \lesssim \mu^{-j-\frac{|\beta|}{2}}
$$

since $R \leq \mu^{\frac{1}{2}}$. By homogeneity, we write

$$
\varphi_{1}(0, \eta)=\left\langle\left(d_{\eta} \varphi_{1}\right)\left(0, \omega_{\nu}\right), \eta\right\rangle+R^{\nu}(0, \eta),
$$

where

$$
R^{\nu}(0, \eta)=\sum_{|\alpha|=2} \int_{0}^{1}\left(\eta-|\eta| \omega_{\nu}\right)^{\alpha} \partial_{\eta}^{\alpha} \varphi_{1}\left(0, s \eta+(1-s)|\eta| \omega_{\nu}\right) d s .
$$

By (5.9), Lemma 5.1, and the inverse function theorem, this yields the following estimates over $\operatorname{supp}\left(a^{\nu}(0, \cdot)\right)$ :

$$
\left|\left\langle\omega_{\nu}, \partial_{\eta}\right\rangle^{j} \partial_{\eta}^{\beta} R^{\nu}(0, \eta)\right| \lesssim \mu^{-j-\frac{|\beta|}{2}} .
$$

Set $x_{\nu}=\bar{x}_{1}\left(0, \omega_{\nu}\right)=d_{\eta} \varphi_{1}\left(0, \omega_{\nu}\right)$. This allows us to write

$$
\langle y, \eta\rangle-\varphi_{1}(0, \eta)=\left\langle y-x_{\nu}, \eta\right\rangle-R^{\nu}(0, \eta)
$$

so that integration by parts gives

$$
\left|\int e^{i\left\langle y-x_{\nu}, \eta\right\rangle}\left(e^{-i R^{\nu}(0, \eta)} a^{\nu}(z, \eta)\right) d \eta\right| \lesssim \mu^{\frac{n+1}{2}}\left(1+\mu\left|\left\langle\omega_{\nu}, y-x_{\nu}\right\rangle\right|+\mu\left|y-x_{\nu}\right|^{2}\right)^{-N} .
$$

Taking the sum over all $\nu$ gives

$$
\left|K_{\varepsilon}(y ; 1,0)\right| \leq C_{N} \sum_{\nu} \mu^{\frac{n+1}{2}}\left(1+\mu\left|\left\langle\omega_{\nu}, y-x_{\nu}\right\rangle\right|+\mu\left|y-x_{\nu}\right|^{2}\right)^{-N} .
$$


We now set $\widetilde{\Omega}_{-}=\Omega_{2}^{-}$. By (5.18), the distance between the complement of $\Omega_{2}^{-}$and $\Lambda_{1}^{-}$is uniformly bounded from below, and (4.17) is straightforward when either $y \notin \Omega_{2}^{-}$or $d\left(y, \Lambda^{-}\right) \geq \tilde{c}_{0}$. Hence from now on we assume $y \in \Omega_{2}^{-}$and $d\left(y, \Lambda^{-}\right) \leq \tilde{c}_{0}$.

Given $\nu$, set $\tilde{\omega}_{\nu}=d_{z} \varphi_{1}\left(0, \omega_{\nu}\right) /\left|d_{z} \varphi_{1}\left(0, \omega_{\nu}\right)\right|$ so that $x_{\nu}=x_{0,1}\left(0, \tilde{\omega}_{\nu}\right)$ and $\omega_{\nu}=$ $\xi_{0,1}\left(0, \tilde{\omega}_{\nu}\right) /\left|\xi_{0,1}\left(0, \tilde{\omega}_{\nu}\right)\right|$, with $\left(x_{0,1}(x, \omega), \xi_{0,1}(x, \omega)\right)$ denoting the solution to Hamilton's equations (5.1) at time 0 with initial conditions $(x, \omega)$ at time 1 . Since

$$
\omega \mapsto x_{0,1}(0, \omega) \quad \text { and } \quad \omega \mapsto \xi_{0,1}(0, \omega) /\left|\xi_{0,1}(0, \omega)\right|
$$

are both bilipschitz maps from $\mathbb{S}^{n-1}$ onto their image, we have that

$$
\left|x_{\nu}-x_{\nu^{\prime}}\right| \approx\left|\omega_{\nu}-\omega_{\nu^{\prime}}\right| \text {. }
$$

Now let $x_{0} \in \Lambda_{z}$ be such that $d\left(y, \Lambda_{z}\right)=\left|y-x_{0}\right|$. Let $\omega_{0}$ be the unit normal to $\Lambda_{z}$ at $x_{0}$ with $\left(\omega_{0}\right)_{1}<0$. As in Lemma 5.2 , we can choose $c_{0}, \tilde{c}_{0}$ small so that there exists $\tilde{\omega}_{0} \in \Omega_{3}$ with $\omega_{0}=\xi_{0,1}(x, \tilde{\omega}) /\left|\xi_{0,1}(x, \tilde{\omega})\right|$ and $\left|\left\langle\omega_{0}, y-x_{0}\right\rangle\right|=d\left(y, \Lambda_{z}\right)$. We claim that

$$
\left|\left\langle\omega_{0}, y-x_{0}\right\rangle\right|+\left|\omega_{0}-\omega_{\nu}\right|^{2} \lesssim\left|\left\langle\omega_{\nu}, y-x_{\nu}\right\rangle\right|+\left|y-x_{\nu}\right|^{2} .
$$

First observe that

$\left|y-x_{0}\right|^{2}+\left|x_{0}-x_{\nu}\right|^{2}=\left|y-x_{\nu}\right|^{2}+2\left\langle y-x_{0}, x_{0}-y\right\rangle+2\left\langle y-x_{0}, y-x_{\nu}\right\rangle \leq 3\left|y-x_{\nu}\right|^{2}$,

and hence

$$
\left|y-x_{\nu}\right|^{2} \approx\left|y-x_{0}\right|^{2}+\left|x_{0}-x_{\nu}\right|^{2} \approx\left|y-x_{0}\right|^{2}+\left|\omega_{0}-\omega_{\nu}\right|^{2} .
$$

Now observe that

$$
x_{\nu}-x_{0}=\frac{\partial x_{0,1}}{\partial \omega}\left(0, \tilde{\omega}_{0}\right) \cdot\left(\tilde{\omega}_{\nu}-\tilde{\omega}_{0}\right)+E,
$$

where $E$ denotes the second order error in the Taylor expansion of $\omega \mapsto x_{0,1}(0, \omega)$ about $\omega_{0}$. Since $\omega_{0} \cdot \frac{\partial x_{0,1}}{\partial \omega}\left(0, \tilde{\omega}_{0}\right)=0$ we have that

$$
\left|\left\langle\omega_{0}, x_{\nu}-x_{0}\right\rangle\right| \lesssim\left|\tilde{\omega}_{\nu}-\tilde{\omega}_{0}\right|^{2} \lesssim\left|\omega_{\nu}-\omega_{0}\right|^{2} .
$$

The claim now follows from the following inequality:

$$
\begin{aligned}
||\left\langle\omega_{0}, y-x_{0}\right\rangle|-|\left\langle\omega_{\nu}, y-x_{\nu}\right\rangle|| & \lesssim\left|\left\langle\omega_{0}-\omega_{\nu}, y-x_{\nu}\right\rangle\right|+\left|\left\langle\omega_{0}, x_{\nu}-x_{0}\right\rangle\right| \\
& \lesssim\left|y-x_{\nu}\right|^{2}+\left|\omega_{0}-\omega_{\nu}\right|^{2} .
\end{aligned}
$$

Since $\left|\left\langle\omega_{0}, y-x_{0}\right\rangle\right|=d\left(y, \Lambda_{z}\right)$, we can now use (5.22) to control the sum in (5.21) to prove the lemma.

\section{ACKNowledgement}

The author would like to thank Professor Hart Smith for suggesting the problem and for his insight on squarefunction and spectral cluster estimates. In addition, the author is grateful to the referee for several helpful comments and suggestions. 


\section{REFERENCES}

[1] Bahouri, H.; Chemin, J.-Y. Equations d'ondes quasilineares et effet dispersif. Int. Math. Res. Not. 1999, 21, 1141-1178. MR.1728676 (2000i:35125)

[2] Bahouri, H.; Chemin, J.-Y. Equations d'ondes quasilineares et estimations de Strichartz. Amer. J. Math. 1999, 121 (6), 1337-1377. MR1719798(2000i:35124)

[3] Blair, M. Strichartz estimates for wave equations with coefficients of Sobolev regularity. Comm. Partial Differential Equations 2006, 31 (5), 649-688. MR.2233036

[4] Cordoba, A.; Fefferman. C. Wave packets and Fourier integral operators. Comm. Partial Differential Equations 1978, 3 (11), 979-1005. MR.507783 (80a:35117)

[5] Koch, H.; Smith, H.; Tataru, D. Sharp $L^{q}$ bounds on spectral clusters for Hölder metrics. Math. Res. Lett. 2007, 14 (1), 77-86. MR2289621 (2008a:35048)

[6] Mockenhaupt, G.; Seeger, A.; Sogge, C. D. Local smoothing of Fourier integrals and CarlesonSjölin estimates. J. Amer. Math. Soc. 1993, 6, 65-130. MR1168960 (93h:58150)

[7] Saloff-Coste, L. Uniformly elliptic operators on Riemannian manifolds. J. Differential Geom. 1992, 36, 417-450. MR 1180389 (93m:58122)

[8] Smith, H. A parametrix construction for wave equations with $C^{1,1}$ coefficients. Ann. Inst. Fourier (Grenoble) 1998, 48 (3), 797-835. MR1644105 (99h:35119)

[9] Smith, H. Sharp $L^{2} \rightarrow L^{q}$ bounds on spectral projectors for low regularity metrics. Math. Res. Lett. 2006, 13 (6), 967-974. MR2280790

[10] Smith, H. Spectral cluster estimates for $C^{1,1}$ metrics. Amer. J. Math. 2006, 128 (5), 10691103. MR2262171

[11] Smith, H.; Sogge, C. On Strichartz and eigenfunction estimates for low regularity metrics. Math. Res. Lett. 1994, 1 (6), 729-737. MR.1306017 (95h:35156)

[12] Smith, H.; Tataru, D. Sharp counterexamples for Strichartz estimates for low regularity metrics. Math. Res. Lett. 2002, 9 (2-3), 199-204. MR1909638 (2003e:35173)

[13] Sogge, C. D. Concerning the $L^{p}$ norm of spectral clusters for second-order elliptic operators on compact manifolds. J. Funct. Anal. 1988, 77 (1), 123-138. MR930395 (89d:35131)

[14] Stein, E. M. Singular Integrals and Differentiability Properties of Functions; Princeton University Press: Princeton, N.J., 1970. MR 0290095 (44:7280)

[15] Tataru, D. Strichartz estimates for second order hyperbolic operators with nonsmooth coefficients and the nonlinear wave equation. Amer. J. Math 2000, 122 (2), 349-376. MR1749052 (2001c:35260)

[16] Tataru, D. Strichartz estimates for second order hyperbolic operators with nonsmooth coefficients II. Amer. J. Math 2001, 123 (3), 385-423. MR1833146 (2002d:35140)

[17] Tataru, D. Strichartz estimates for second order hyperbolic operators with nonsmooth coefficients III. J. Amer. Math. Soc. 2002, 15 (2), 419-442. MR.1887639 (2003a:35120)

[18] Taylor, M.E. Partial Differential Equations. III; Applied Mathematical Sciences 117, Springer-Verlag: New York, 1996. MR.1477408 (98k:35001)

[19] Taylor, M.E. Pseudodifferential Operators and Nonlinear PDE; Birkhhäuser: Boston, 1991. MR.1121019 (92j:35193)

[20] Taylor, M.E. Tools for PDE. Pseudodifferential Operators, Paradifferential Operators, and Layer Potentials; Mathematical Surveys and Monographs, Vol. 81, American Mathematical Society: Providence, R.I., 2000. MR.1766415 (2001g:35004)

Department of Mathematics, University of Rochester, Rochester, New York 14627 E-mail address: blair@math.rochester.edu

Current address: Department of Mathematics and Statistics, University of New Mexico, Albuquerque, New Mexico 87131

E-mail address: blair@math.unm.edu 